\title{
A confined dynamo: magnetic activity of the K-dwarf component in the pre-cataclysmic binary system V471 Tauri
}

\author{
Zs. Kôvári ${ }^{1}$, L. Kriskovics ${ }^{1 \star}$, K. Oláh ${ }^{1}$, P. Odert ${ }^{2,3}$, M. Leitzinger ${ }^{2,3}$, B. Seli ${ }^{1}$, K. Vida $^{1}$, T. Borkovits ${ }^{1,4}$, and T. Carroll ${ }^{5}$ \\ ${ }^{1}$ Konkoly Observatory, Research Centre for Astronomy and Earth Sciences, Konkoly Thege út 15-17., H-1121 Budapest, Hungary \\ e-mail: kovari@konkoly.hu \\ 2 Institute of Physics/IGAM, University of Graz, Universitätsplatz 5, A-8010 Graz, Austria \\ 3 Space Research Institute, Austrian Academy of Sciences, Schmiedlstraße 6, A-8042 Graz, Austria \\ 4 Baja Astronomical Observatory of University of Szeged, Szegedi út, Kt. 766, H-6500 Baja, Hungary \\ 5 Leibniz-Institute for Astrophysics (AIP), An der Sternwarte 16, D-14482 Potsdam, Germany \\ Received ...; accepted ...
}

\begin{abstract}
Context. Late-type stars in close binary systems can exhibit strong magnetic activity owing to rapid rotation supported by tidal locking. On the other hand, tidal coupling may suppress the differential rotation which is a key ingredient of the magnetic dynamo. Aims. We scrutinize the red dwarf component in the eclipsing binary system V471 Tau in order to unravel relations between different activity layers from the stellar surface through the chromosphere up to the corona. We aim at studying how the magnetic dynamo in the late-type component is affected by the close white dwarf companion.

Methods. We use space photometry, high resolution spectroscopy and X-ray observations from different space instruments to explore the main characteristics of magnetic activity. We apply a light curve synthesis program to extract the eclipsing binary model and further analyze the residual light variations. Photometric periods are obtained using a Fourier-based period search code. We search for flares by applying an automated flare detection code. Spectral synthesis is used to derive or specify some of the astrophysical parameters. Doppler imaging is used to reconstruct surface temperature maps, which are cross-correlated with each other to derive surface differential rotation. We apply different conversion techniques to make it possible to compare the X-ray emissions obtained from different space instruments.

Results. From $K 2$ photomery we find that 5-10 per cent of the apparent surface of the red dwarf is covered by cool starspots. From seasonal photometric period changes we estimate a weak differential rotation. From the flare activity we derive a cumulative flare frequency diagram which suggests that frequent flaring could have a significant role in heating the corona. Using high resolution spectroscopy we reconstruct four Doppler images for different epochs which reveal an active longitude, that is, a permanent dominant spot facing the white dwarf. From short term changes in the consecutive Doppler images we derive a weak solar-type surface differential rotation with $\alpha_{\mathrm{DR}}=0.0026$ shear coefficient, similar to that provided by photometry. The long-term evolution of X-ray luminosity reveals a possible activity cycle length of $\approx 12.7 \mathrm{ys}$, traces of which were discovered also in the $\mathrm{H} \alpha$ spectra.

Conclusions. We conclude that the magnetic activity of the red dwarf component in V471 Tau is strongly influenced by the close white dwarf companion. We confirm the presence of a permanent dominant spot (active longitude) on the red dwarf facing the white dwarf. The weak differential rotation of the red dwarf is very likely the result of tidal confinement by the companion. We find that the periodic appearance of the inter-binary $\mathrm{H} \alpha$ emission from the vicinity of the inner Lagrangian point is correlated with the activity cycle.
\end{abstract}

Key words. stars: activity - stars: late-type - stars: imaging - stars: starspots - Stars: individual: V471 Tau

\section{Introduction}

Magnetic fields have a strong effect on stellar structure and overall, on the long-term evolution of stars, including our Sun. Studying the manifestations of stellar magnetic activity, from photospheric starspots through the bright chromospheric features to the active corona, helps us understand the nature of the underlying magnetic dynamo, and eventually the magnetic evolution of stars. In the last few years it became feasible to study flares on a large number of stars with the Kepler, K2 and ongoing TESS missions (e.g. Balona 2015). Such continuous high-precision space photometry combined with high-resolution spectroscopic observations can reveal the connection between the occurrence rate of flares and spot distributions.

\footnotetext{
^ Bolyai János Research Fellow
}

It has been learned that late-type stars in close binary systems can exhibit even stronger magnetic activity owing to rapid rotation supported by tidal locking (e.g. Hill et al. 2014). Tidal coupling, however, may suppress the differential rotation (Scharlemann 1982) which is a key ingredient of the magnetic dynamo. In the recent study by Kövári et al. (2017) it was shown that the tidal effect of a close companion star has indeed a suppressive effect on differential rotation.

In our study we scrutinize the red dwarf component in the eclipsing binary system V471 Tau in order to explore relations between different activity layers from the stellar surface through the chromosphere up to the corona. V471 Tau appeared first in the literature as a spectroscopic binary in the General Catalogue of Stellar Radial Velocities (GCVR, Wilson 1953). The system located in the Hyades star cluster consists of DA white dwarf primary with a $\mathrm{K} 2 \mathrm{~V}$ companion, forming a post-common enve- 
lope binary (PCEB) star. The age of the Hyades cluster of $t \approx$ $680 \mathrm{Myr}$ provides an upper limit for the age of V471 Tau (Gossage et al. 2018). The binary is dubbed to be a pre-cataclysmic variable as well, which means that there is no significant mass transfer in the system, because the K star does not fill its Roche lobe. The orbital period of the eclipsing system of $0.52118 \mathrm{~d}$ (for updated orbital parameters see Vaccaro et al. 2015) is modulated on the long term, which was explained by a light-time effect due to the gravitational influence of a third body, possibly a brown dwarf (Guinan \& Ribas 2001). As an alternative explanation, the Applegate mechanism was offered by Hardy et al. (2015), where eclipse timing variations were interpreted as changes in the quadrupole moment within the K2V star. Recently, Lanza (2020) has proposed a mechanism based on a permanent non-axisymmetric gravitational quadrupole moment. It is no exaggeration to say that V471 Tau is an actual astrophysical laboratory for studying many aspects of stellar evolution. The white dwarf primary with a surface temperature of $\approx 35000 \mathrm{~K}$ has strong emission in the ultraviolet (UV), extreme ultraviolet (EUV) and X-ray regimes. In such PCEB systems X-ray eemission can originate from either the white dwarf or the corona of the K star. Moreover, the hot primary can also heat the exposed hemisphere of the secondary. V471 Tau is a well-known variable at the high energy electromagnetic regime as well, being observed during the Einstein (Young et al. 1983), IUE (Guinan \& Sion 1984), EXOSAT Jensen et al. (1986), ROSAT (Barstow et al. 1992; Wheatley 1998) and more recently in the course of Chandra (García-Alvarez et al. 2005) and XMM Newton missions, to mention the most important ones. Accordingly, among other major findings, Jensen et al. (1986) discovered of the pulsation of the white dwarf, while (Barstow et al. 1992) confirmed a weak accretion from the $\mathrm{K}$ star to the white dwarf via stellar wind. Using $U V$ data from Goddard High Resolution Spectrograph (GHRS) on board the Hubble Space Telescope Bond et al. (2001) reported Coronal Mass Ejections (CMEs) from the active $\mathrm{K}$ dwarf and estimated a 100-500 CMEs per day emission rate, i.e. about a hundred times higher frequency compared to the Sun.

As for the surface magnetic activity of the secondary, the $\mathrm{K} 2 \mathrm{~V}$ star, forced to rotate synchronously with the orbital period, is known to exhibit rotational variability due to starspots (e.g. Evren et al. 1986) and flare activity (Young et al. 1983). The first Doppler images of the star were presented by Ramseyer et al. (1995), who obtained four separate temperature maps spanning over a year. In another Doppler-imaging study Hussain et al. (2006) found that despite the tidally inhibited differential rotation the K-dwarf component of the V471 Tau binary system showed indeed a high level of magnetic activity. In our paper we perform a complex study of the magnetic activity of V471 Tau, including new Doppler images and flare statistics for the spotted red dwarf.

The paper is organized as follows. In Sect.2 we summarize the available photometric and spectroscopic data to be analyzed. Using Kepler $K 2$ observations, in Sect. 4 by extracting orbital solution we analyze light variations due to spots and flares. In Sect. 3 by the means of spectral synthesis we derive precise astrophysical parameters of the active K-dwarf component, which is used to perform a Dopper imaging study in Sect.5 where we estimate the surface differential rotation of the spotted star. The chromospheric and coronal activity of the $\mathrm{K}$ star is analyzed in Sect.6.2. Our results are discussed in Sect.7 and summarized in Sect.8.

\section{Data}

\subsection{K2 photometry}

The Kepler K2 mission provided long-term high-precision space photometry (Howell et al. 2014) of V471 Tau (EPIC 210619926). The star was observed as part of the 4th Campaign of the mission between 08 February-20 April 2015 (BJD 2457061.7910-2457132.6877), covering 136 subsequent orbital cycles. Both short (1-min) and long cadence (30-min) light curves of V471 Tau are available for the interval. Although the $K 2$ data do not coincide with the spectroscopic observations used in this paper, the short cadence space data are suitable for studying spot evolution and flare activity on a somewhat longer timescale. As an example, in Fig.11 we plot a two cycles long part of the short cadence light curve, where in-eclipse and out-of-eclipse flares are also seen.

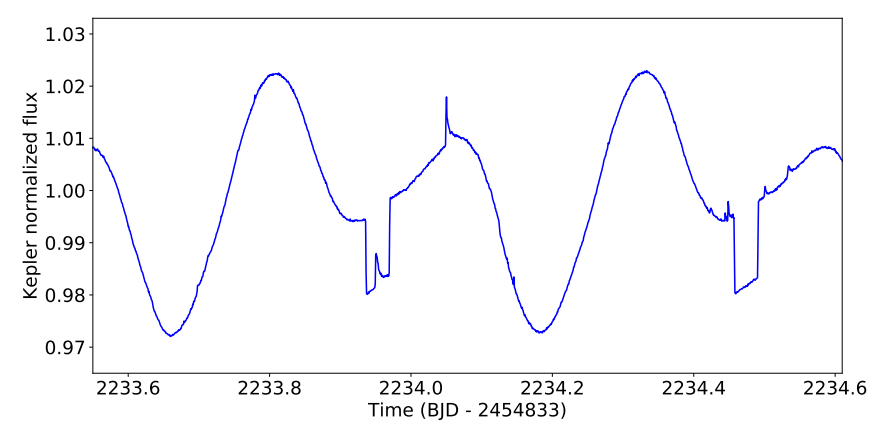

Fig. 1. Part of the Kepler K2 short cadence data of V471 Tau with a particular flare event occured when the white dwarf component was in eclipse.

\subsection{High-resolution spectroscopy}

We use high-resolution $(R=81000)$ spectroscopic observations of V471 Tau publicly available in the data archive of the Echelle SpectroPolarimetric Device for the Observation of Stars (ESPaDOnS) at Canada-France-Hawaii Telescope (CFHT). The spectra cover the 3700-10500 $\AA$ optical range. The available data were retrieved in two seasons, the first in December 2005 and the second at the turn 2014/2015. We selected 297 and 222 spectra from the two seasons, respectively, to achieve uttermost subsets (in the long run two in both seasons) with regard to optimal phase coverage for Doppler imaging (see Sect. 5]. Low quality spectra with signal-to-noise $\mathrm{S} / \mathrm{N}<40$ were omitted from the selection. Before using the four data subsets for Doppler image reconstructions we shifted the spectra to the rest wavelength and continuum fitting was carried out. The CFHT spectra contain the $\mathrm{H} \alpha$ region which is used to study the chromospheric activity and discover possible mass motions in the binary (see Sec.6.1). The observing log of the CFHT-ESPaDOnS spectra used in our study is given in Table A.1 in Appendix A

\subsection{X-ray observations}

The V471 Tau system has been detected with many X-ray instruments during the last decades. Here we attempt to infer the longterm variability of the $\mathrm{K}$ dwarf's coronal emission. As none of the X-ray observations coincide with the epochs of the high resolution spectroscopic data for Doppler imaging, we do not address its variability on shorter timescales like rotational modulation 
due to the distribution of active regions. We note that the binary components are not spatially resolved in the X-ray observations, but it is well established that only the softer $(>50 \AA)$ wavelength range is affected by the emission from the white dwarf (Drake \& Sarna 2003; García-Alvarez et al. 2005), therefore its eclipse (at $\phi=0$ orbital phase) is only observed in soft X-ray/EUV light curves (Cully et al. 1996, Wheatley 1998). What makes the investigation of the long-term coronal variability difficult is that V471 Tau was rarely observed by the same instrument more than once, the only exception being the ROSAT pointed survey (twice during the same year) and XMM Newton (separated by 15 years). Moreover, the $\mathrm{K}$ dwarf is very active due to its high rotation rate, so its emission is variable also on short time scales, including frequent flares, which makes the definition of a quasi-quiescent emission level during a given epoch difficult.

For the analysis we select data from five X-ray instruments covering the years 1991-2019 (Table 1). We do not include data from earlier instruments (Exosat, Einstein) and the ROSAT All Sky Survey (RASS), because of their softer wavelength coverage (including mainly the white dwarf's emission) and/or their short duration (RASS). When selecting the space data we considered the epochs of our new Doppler reconstructions as well (see Sect.5). In Sect.6.2 we describe the detailed analysis steps for each instrument and observation, as well as which data were taken from previous analyses in the literature.

\section{Stellar properties}

\subsection{Adopted parameters}

The Gaia DR2 parallax of $\pi=20.957 \pm 0.044$ mas (Gaia Collaboration et al.2018) yields a distance of $d=47.72 \pm 0.10 \mathrm{pc}$ for V471 Tau. This distance assuming $V_{\mathrm{br}} \approx 9 \mathrm{~m} .35$ for the brightest $V$ magnitude observed so far in 2004 (cf. İbanoğlu et al. 2005) and removing $\Delta V_{\mathrm{wd}}=-0.02$ as the contribution of the white dwarf (Rucinski 1981) and taking into account a maximum of $A_{V}=0.003$ insterstellar exctinction (cf. Taylor 2006) yields an absolute visual magnitude $M_{V}=5 \mathrm{~m} .97 \pm 0$ m.03. Taking $B C=-0.257$ bolometric correction from (Flower 1996) gives a bolometric magnitude $M_{\mathrm{bol}}=5 \mathrm{~m} \cdot 72 \pm 0 \mathrm{~m} \cdot 03$. This gives $L / L_{\odot}=0.41 \pm 0.01$ when using $M_{\text {bol, } \odot}=4.74$ value for the Sun. On the other hand, when adopting $R / R_{\odot}=0.91 \pm 0.02$ (Vaccaro et al. 2015, see their Table 11) and with $T_{\text {eff }}=4980 \mathrm{~K}$ (see Sect. 3.2) the Stefan-Boltzmann law would give a bit higher $L / L_{\odot}$ ratio of $0.46 \pm 0.04$; still in a fair agreement with the value obtained from the bolometric magnitude.

We perform a spectral energy distribution (SED) synthesis using the virtual observatory (VO) tool VOSA (Bayo et al. 2008) to build the SED of V471 Tau from the available VO catalogs. We use VOSA to collect archival photometry from $T y$ cho, SLOAN/SDSS, Gaia DR2, 2MASS, AKARI/IRC, and WISE surveys. As input we confined the effective temperature to $4950 \mathrm{~K}<T_{\text {eff }}<5050 \mathrm{~K}$, the surface gravity to $4.25<\log g<4.75$, and the metallicity to $-0.2<[\mathrm{Fe} / \mathrm{H}]<0.2$ (cf. Sect. 3.2). The resulting synthetic spectrum plotted in Fig. 2 corresponds to $L / L_{\odot}$ of $0.40 \pm 0.21$, in agreement with both values given hereinabove.

The $v \sin i=91 \mathrm{~km} \mathrm{~s}^{-1}$ projected rotational velocity of the K2 star was adopted from Hussain et al. (2006, see also their references). The inclination $i=77: 4_{-4.5}^{+1.5}$ of the binary orbit with respect to the line of sight was derived in O'Brien et al. (2001), which we adopt here for the K-dwarf with the assumption that its rotational axis is perpendicular to the orbital plane.

Adopting $P_{\text {orb }}=P_{\text {rot }}$ period and $\mathrm{HJD}_{0}=2440610.06406$ reference time (mid-primary minimum, when the white dwarf is in eclipse) from Guinan \& Ribas (2001) we use the following equation for calculating the phase values of the spectroscopic observations in Table A.1.

$\mathrm{HJD}_{\phi=0}=2440610.06406+0.521183398 \times E$.

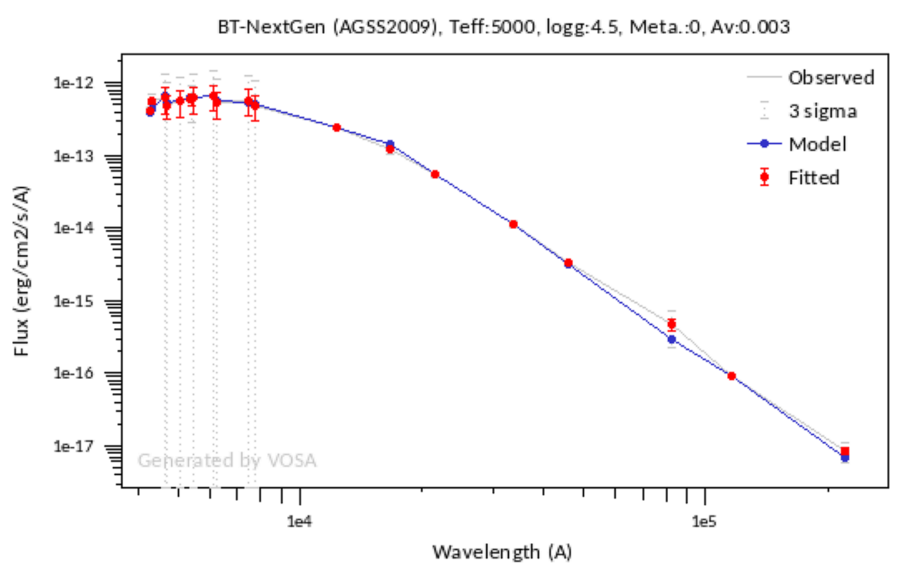

Fig. 2. Spectral energy distribution for V471 Tau generated by the VOSA SED analyzer tool (Bayo et al. 2008). The synthetic spectrum (blue line) is fitted to the available archival photometry (red dots) from Tycho, SLOAN/SDSS, Gaia DR2, 2MASS, AKARI/IRC, and WISE surveys.

\subsection{Spectral synthesis}

We carried out a detailed spectroscopic study based on spectral synthesis using the code SME (Piskunov \& Valenti 2017). During the synthesis, MARCS models were used (Gustafsson et al. 2008) and atomic paramaters were taken from VALD (Kupka et al. 1999). Macroturbulence was computed using the following equation from Valenti \& Fischer (2005):

$v_{\text {mac }}\left[\mathrm{km} \mathrm{s}^{-1}\right]=3.98-\frac{T_{\text {eff }}[K]-5770}{650}$.

First, we selected 15 good quality spectra observed in eclipses, when only the $\mathrm{K} 2$ star was seen, and performed the synthesis independently for the 15 spectra to get $T_{\text {eff }}, \log g$, and $[\mathrm{Fe} / \mathrm{H}]$. We used the initial values as input for subsequent iterations. We gradually confined the microturbulence and the projected rotational velocity at $v_{\text {mic }}=0.7( \pm 0.2) \mathrm{km} \mathrm{s}^{-1}$ and $v \sin i=91( \pm 1) \mathrm{km} \mathrm{s}^{-1}$, respectively, in agreement also with literary values (cf., e.g., Hussain et al.2006; Adibekyan et al.2012, and their references). Finally, the surface gravity was kept fixed at $\log g=4.5$, in agreement with former, well established values (see Vaccaro et al. 2015, and their references). At the end, the procedure resulted in $T_{\text {eff }}=4980 \mathrm{~K}$ and $[\mathrm{Fe} / \mathrm{H}]=0.12$. For details of the iterative fitting method see Kriskovics et al. (2019). The final astrophysical parameters with their errors are listed in Table 2.

In the second test we run the synthesis for all the available CFHT spectra to see if the surface temperature varies along the orbital phase. Therefore, all of the previously derived parameters were kept fixed except for the surface temperature. The results are plotted in Fig. 3, demonstrating that the surface of the red dwarf is indeed the least affected around $\phi=0.0$ when the hot component is obscured. The temperature rise of $\approx 100 \mathrm{~K}$ at $\phi=0.5$ compared to the eclipse either in 2005 (blue dots in the figure) or 2014/15 (red dots) may be due to the irradiation of 
Table 1. Adopted X-ray observations of V471 Tau with their durations and the quiescent periods within them

\begin{tabular}{ccccrr}
\hline Date [dd.mm.yyyy] & Mission & Instrument & Observing ID & Duration [s] & Quiet [s] \\
\hline \hline 23.08 .1991 & ROSAT & PSPC & RP200107M01 & 27692 & all \\
26.08 .1996 & ASCA & SIS+GIS & 24032000 & 102416 & all \\
14.08 .1997 & ROSAT & HRI & RH202309N00 & 15793 & 1104 \\
24.01 .2002 & Chandra & HRC-S/LETG & 2523 & 87990 & all \\
01.08 .2004 & XMM Newton & EPIC/PN & 0203260101 & 60924 & 18783 \\
30.07 .2015 & Swift & XRT & 33909 & 2447 & all \\
04.09 .2019 & XMM Newton & EPIC/PN & 0844350101 & 63500 & 9381 \\
\hline
\end{tabular}

the hot component, but surface activity can also play a role (see Sect.7.2 for a discussion). Indeed, it is very likely that the stellar dynamo in the synchronously rotating red dwarf is affected by the close companion and therefore manifestations of magnetic activity may be coupled to the orbital phase (cf. Schrijver \& Zwaan 1991).

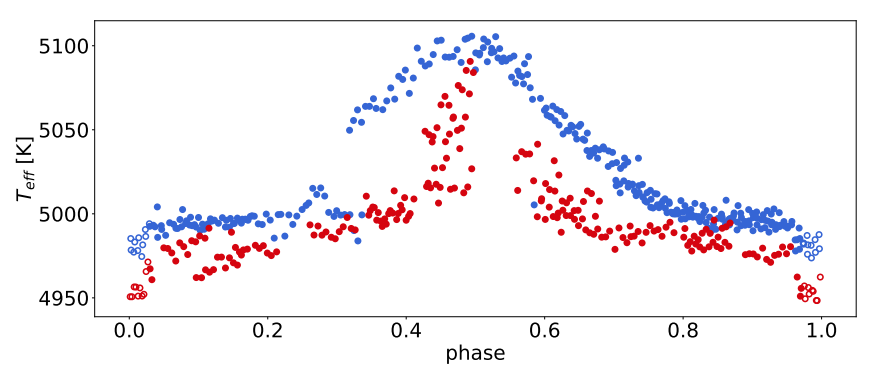

Fig. 3. Variation of the surface temperature of the $\mathrm{K} 2$ star along the orbital phase. Dots show the temperature values derived individually for each spectra (blue for the 2005 season, red for the 2014/15 spectra) by spectral synthesis. Open circles around zero phase correspond to those unaffected spectra that were observed when the white dwarf was eclipsed. The temperature rise of $\approx 100 \mathrm{~K}$ at $\phi \approx 0.5$ in both seasons may indicate the irradiation effect of the hot component. See Sect. 3.2 for details.

Table 2. Fundamental astrophysical data of the K2 dwarf component of V471 Tau

\begin{tabular}{ll}
\hline Parameter & Value \\
\hline \hline Spectral type & K2 V \\
Gaia distance [pc] & $47.72 \pm 0.10$ \\
$V_{\text {br }}[\mathrm{mag}]$ & $9 \mathrm{~m} 37 \pm 0.03$ \\
$M_{\text {bol }}[\mathrm{mag}]$ & $5.72 \pm 0.03$ \\
Luminosity $\left[L_{\odot}\right]$ & $0.41 \pm 0.01$ \\
$T_{\text {eff }}[\mathrm{K}]$ & $4980 \pm 10$ \\
$\log g($ in cgs $)$ & $4.50 \pm 0.05$ \\
{$[\mathrm{Fe} / \mathrm{H}]$} & $0.12 \pm 0.04$ \\
$v_{\text {mic }}\left[\mathrm{km} \mathrm{s}^{-1}\right]$ & $0.7 \pm 0.2$ \\
$v_{\text {mac }}{ }^{a}\left[\mathrm{~km} \mathrm{~s}^{-1}\right]$ & 5.2 \\
$v$ sin $i\left[\mathrm{~km} \mathrm{~s}^{-1}\right]$ & $91 \pm 1$ \\
Rotation period $[\mathrm{d}]$ & 0.521183398 \\
Inclination $^{b}\left[{ }^{\circ}\right]$ & $77.5_{-4.5}^{+7.5}$ \\
Radius $^{c}\left[R_{\odot}\right]$ & $0.91 \pm 0.02$ \\
Mass $^{c}\left[M_{\odot}\right]$ & $0.95 \pm 0.05$ \\
\hline
\end{tabular}

Notes. ${ }^{(a)}$ Computed using Eq.2 ${ }^{(b)}$ O'Brien et al. (2001); ${ }^{(c)}$ Vaccaro et al. (2015).

Article number, page 4 of 21

\section{Photometric analysis}

\subsection{Extracting the eclipsing binary light curve}

Before analyzing the photometric signs of the magnetic activity of V471 Tau we removed the light curve variations caused by the close binary nature as follows. First, we formed a phasefolded light curve with the use of the $K 2$ short cadence light curve. The data were grouped into 2000 orbital phase bins of equal duration. Then, the average of each phase cells were calculated and rendered to the mid-phase point of the given cell. This way the fastest part of the non-orbital phase-locked light variations were removed, but a significant amount of the intrinsic variability over longer timescales has remained in the light curve. In order to remove these slower variations we synthesized a pure binary light curve with the use of the software package Lightcurvefactory (Borkovits et al. 2020). The input parameters were taken from Vaccaro et al.(2015). Besides the eclipses, the binary model has included ellipsoidal variations and the effect of Doppler-boosting, too. We did not consider, however, the reflection/irradiation effect of which the photometric contribution was found to be negligible. Using the parameters from Vaccaro et al. (2015, see their Table 8, 4th column) after a natural readjustment of the mid-time of a given eclipse $\left(T_{0}\right)$ parameter to the value appropriate for $K 2$ data and comparing the synthesized light curve against the folded, binned, averaged $K 2$ light curve, the residual curve has shown significant shoulders around the partial phases of the eclipses, revealing, that the durations of both the total eclipses and the totality should slightly be readjusted. We made it manually with slight modifications of the radius of the $\mathrm{K}$ dwarf component. This way we were able to remove the shoulders from the residual file. Finally, with a repeated use of Lightcurvefactory, this slightly refined synthetic light curve was removed from the original, $\approx 71 \mathrm{~d}$ long $K 2$ measurements. The resulting residual light curve is plotted in Fig. 4

\subsection{Analyzing spot variability}

According to Fig. 4 the light curve is solidly modulated by a continuously changing spot distribution. The relative intensity of the spot amplitude varies between 0.03-0.06. From this we estimate that 5-10 per cent of the apparent stellar surface is covered by spots (see Eq. 3 in Notsu et al. 2019). Assuming surface differential rotation, the change in the rotational frequency signals can be interpreted as a change in the latitude of the dominant surface spots. To recover the presence of differential rotation from the 136-cycle ( $\approx 71$-day) long photometric time series we apply the following technique. First, the entire residual light curve (bottom panel of Fig. 4) was divided into eight consecutive sections of $\approx 9$-day long ( 17 rotational cycles), i.e., a compromise to avoid averaging frequency signals over a longer time scale but still get stable/reliable frequencies for the given part. To derive 

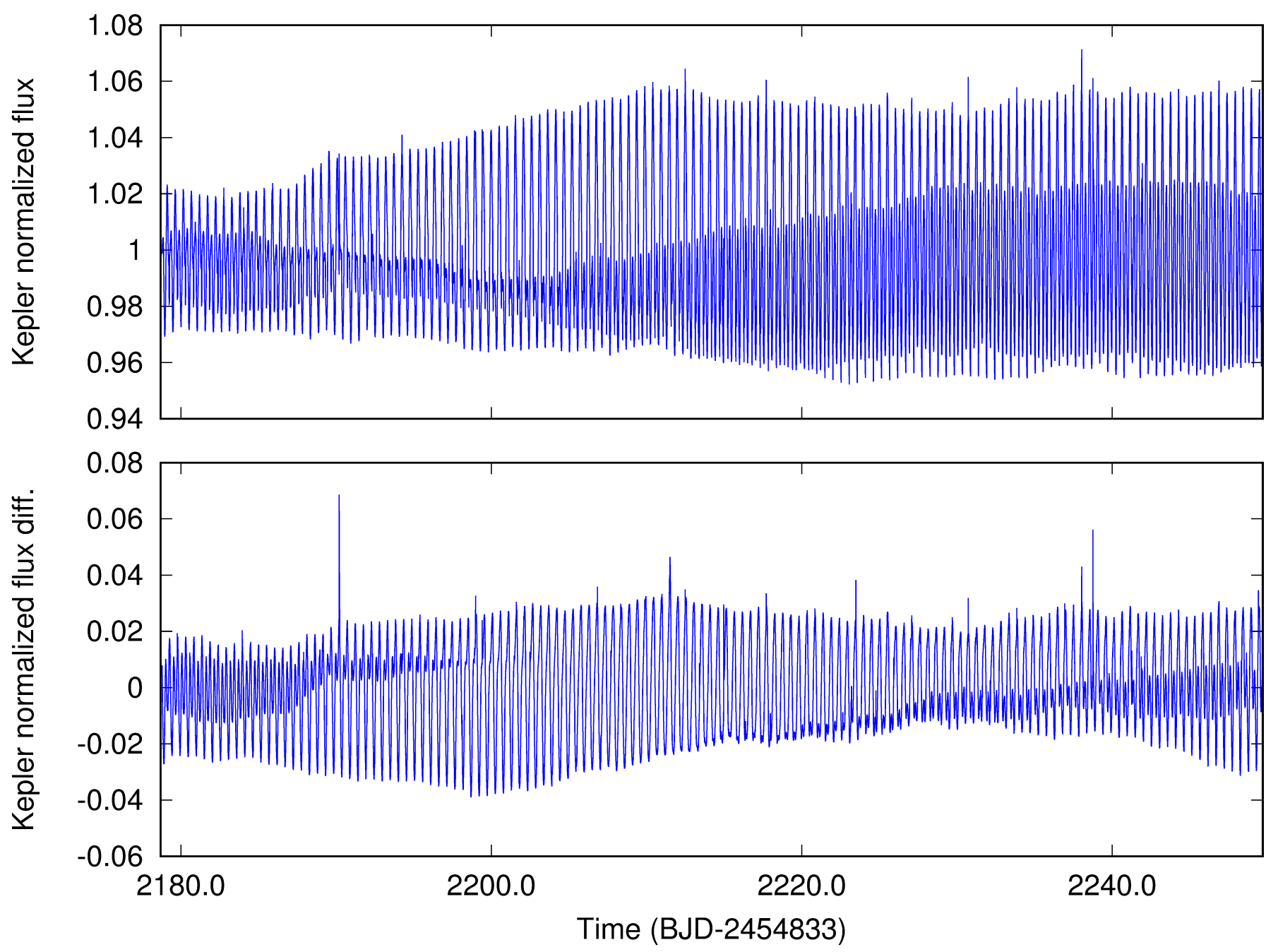

Fig. 4. $K 2$ light curve of V471 Tau (top) and residual light variations (bottom) due to spots and flares after extracting the eclipsing binary model. See Sect.4.1 for details.

the most characteristic rotational period for each part we apply the Fourier-based period search code MuFrAn (Csubry \& Kolláth 2004). The resulting short term rotational periods listed in Table 3 indicate that the change in the period remains within a narrow interval of $\Delta P \approx 0.001$ day. Dividing this by the average rotation period from Table 2, we can estimate the dimensionless surface shear coefficient as $\left|\alpha_{\mathrm{DR}}\right| \gtrsim \Delta P_{\mathrm{phot}} / \bar{P}$, where $\Delta P_{\text {phot }}$ is the full range of the seasonal photometric period associated with surface spots, while $\bar{P}$ is the average period over long term. From the values listed in Table 3, we obtain $\left|\alpha_{\mathrm{DR}}\right| \gtrsim 0.002$, i.e, a weak shear, almost solid-body rotation. We note, that this method alone does not allow to determine the sign of the surface shear parameter, i.e., whether the differential rotation is solar type or antisolar.

\subsection{Finding flares}

After extracting the eclipsing binary model from the $K 2$ light curve we search for flares in residual time series (bottom panel of Fig.4.1) by applying the automated flare detection code FLATW'RM (FLAre deTection With Ransac Method, Vida \& Roettenbacher 2018). The code enables the user to adjust the detection level and the number of successive points associated with a given flare. This is especially important when detecting the
Table 3. Seasonal photometric periods from $K 2$ data

\begin{tabular}{cccc}
\hline $\begin{array}{c}\text { Season } \\
\text { No. }\end{array}$ & \begin{tabular}{c}
$\mathrm{BJD}_{\text {start }^{a}}$ \\
\hline \hline 1
\end{tabular} & $\begin{array}{c}\text { BJD } \\
\text { end }^{a}\end{array}$ & $\begin{array}{c}P_{\text {phot }} \\
{[\mathrm{d}]}\end{array}$ \\
\hline 2 & 2228.790988 & 2236.999957 & 0.520938 \\
3 & 2246.0000241 & 2245.999560 & 0.520565 \\
4 & 2255.000446 & 2263.9999913 & 0.520193 \\
5 & 2264.000594 & 2272.999345 & 0.520336 \\
6 & 2273.000026 & 2281.999446 & 0.519983 \\
7 & 2282.000127 & 2290.999555 & 0.520641 \\
8 & 2291.000236 & 2299.687090 & 0.519987 \\
\hline
\end{tabular}

Notes. ${ }^{(a)}+2454833.0$

smallest events just above the noise level, which are always difficult to find. However, close to the noise limit the number of false positives is starting to increase, which, on the other hand, can be compensated by increasing the number of associated points. After a visual inspection, however, we declared 15 false positives among the detected events. On the other hand, a similar number of possible flares just above the noise level could have been missed. Due to these reasons, we ran a second analysis using a flare-searching neural network (Vida et al., in prep.), and cor- 


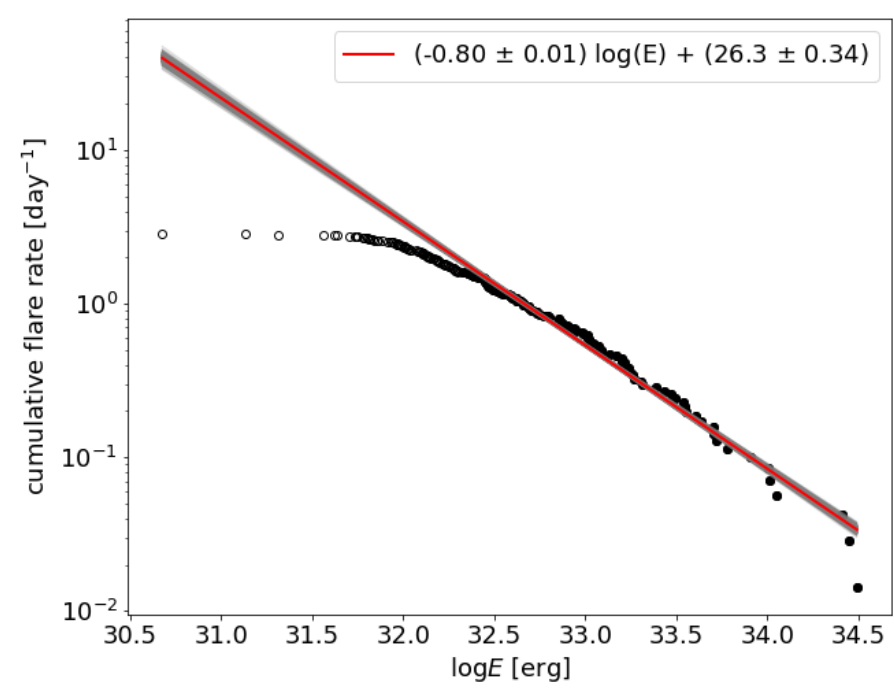

Fig. 5. Cumulative flare-frequency diagram for V471 Tau. The slope of the linear fit (orange line) corresponds to $\alpha=1.8$ power law index.

rected its output manually for possible false positive/negative detections. This analysis yielded a final number of 198 confirmed flare events in the 71-day long $K 2$ time series.

Taking $T_{\text {eff }}$ and $R$ from Table 2 the blackbody approximation yields $L_{\mathrm{q}}=4.64 \times 10^{32} \mathrm{erg} \mathrm{s}^{-1}$ quiescent luminosity for the $\mathrm{K}$ dwarf through the Kepler filter. To determine the individual flare energies, first, the background variation (due to spots) is removed by fitting a low-order polynomial to the 1 hour interval of the given flare (omitting the flaring points). The order of the polynomial is determined by the Bayesian Information Criterion. After subtracting this polynomial, the net flare light curve is integrated over the duration of the flare, yielding the $\varepsilon_{\mathrm{f}}$ relative flare energy (or equivalent duration) of the event. Multiplying $\varepsilon_{\mathrm{f}}$ with $L_{\mathrm{q}}$ gives the total energy $E_{\mathrm{f}}$ released by the flare. Finally, $E_{\mathrm{f}}$ values are used to make up the cumulative flare frequency diagram in Fig. 5

In principle, the cumulative number of flares vs. flare energy can be described by a power law (Gershberg 1972). Accordingly, the logarithm of the $v(E)$ cumulative number of flares with energy values larger than or equal to $E$ can be written as:

$\log v(E)=c_{1}+c_{2} \log E$,

where $c_{1}$ is the intercept while $c_{2}$ is the slope of a linear function. Rewriting the slope $c_{2}=1-\alpha$ one can introduce $\alpha$, that is the power law index of the flare energy distribution (Hawley et al. 2014). The low energy turnover of the diagram below $\log E \approx$ 32.6 [erg] is most probably the result of the detection threshold. Fitting the distribution above this turnover (see the red line in Fig. 5) yields a power law index of $\alpha=1.80 \pm 0.01$. For further discussion about the flare statistics see Sect.7.3.

\section{Doppler imaging}

\subsection{The state-of-the art imaging code iMap}

To reconstruct the surface temperature distribution map of V471 Tau we use the Doppler imaging code iMap (Carroll et al. 2012). This state-of-the-art code carries out multi-line inversion on a list of photospheric lines between 5000-6750 A. We selected 24 non-blended absorption lines with suitable line-depth, temperature sensitivity and well defined continuum. The stellar surface is modelled on a $5^{\circ} \times 5^{\circ}$ resolution grid. Each local line profile is computed with a full radiative solver (Carroll et al. 2008). The local line profiles are disk integrated, and the individually modelled, disk-integrated lines are averaged. Atomic line data are taken from the Vienna Atomic Line Database (VALD Kupka et al. 1999). Model atmospheres are taken from Castelli \& Kurucz (2004) and are interpolated for the necessary temperature, gravity or metallicity values. When solving the radiative transfer local thermodynamical equlibrium (LTE) is assumed because spherical model atmospheres would have required too much computational capacity. Neverheless, imperfections due to the LTE approximation in the fitted line shapes are well compensated by the multi-line approach. Additional input parameters are micro- and macroturbulence, and the projected equatorial velocity (cf. Sect.3).

For the surface reconstructions iMap uses an iterative regularization based on a Landweber algorithm (Carroll et al. 2012). The iterative regularization has been proven to converge always on the same image solution (for the tests see Appendix A in Carroll et al.2012). Therefore, no additional constraints are imposed for the image reconstruction.

\subsection{Surface temperature maps}

In order to achieve good enough phase coverage for the $\approx 0.52$ day rotational period, we decided to form two subsets for both observing seasons. The first two sets for the 2005 season, henceforth S1 and S2, consist of 149 and 172 spectra, respectively, both covering rougly four rotations ( $\approx 2$ days). The S3 and S4 subsets for the 2014/15 season consist of 131 and 97 spectra, respectively, however, due to unfavourable data distribution in this season the $\mathrm{S} 3$ subset covers $\approx 20$ consecutive rotations $(\approx 10$ days) while S4 covers ten rotations (5 days); see Table A.1 in the Appendix. All four subsets are used to reconstruct one Doppler image. The mean HJDs for the four subsets are 2453719.705, $2453722.211,2457016.565$, and 2457031.375, respectively. The four Doppler images (referred hereafter as S1, S2, S3 and S4) presented below, can be regarded as "snapshots" of the stellar surface at these times.

\subsubsection{Doppler images from the 2005 CFHT spectra}

In Fig. 6, the two subsequent Doppler maps for the 2005 season $(\mathrm{S} 1, \mathrm{~S} 2)$ are plotted in orthographic projection in the quadrant phases of the rotation. The corresponding line profile fits are plotted in Figs. A.1 A.2 Both temperature maps show mostly cool spots at similar locations, accounting for the reliability of the image reconstruction. Indeed, most of the cool surface features appear on the combined Doppler map derived from all of the spectra $(\mathrm{S} 1+\mathrm{S} 2)$; see Fig. 7 .

The strongest feature, a cool, high latitude spot on the hemisphere of the visible pole of $\Delta T>-1000 \mathrm{~K}$ temperature contrast relative to $T_{\text {eff }}$ is shown on both $\mathrm{S} 1$ and $\mathrm{S} 2$ maps at around 0.45 phase, centered at $\beta \approx 50^{\circ}$. In addition, this large spot is getting more compact from S1 to S2. A mid-latitude spot is seen on the opposite (lower) hemisphere at $\approx 0.3$ phase. Its location, however, is a bit shifted from S1 to S2 towards the invisible pole, or alternatively, its extension decreases. A weak cool spot is seen on $\mathrm{S} 1$ at $\approx 0.13$ phase, elongated from $30^{\circ}$ latitude down to the equator and even below to $-30^{\circ}$. Such an elongated shape, however, may be the result of mirroring due to the high inclination. In deed, its antitype on S2 is less contrasted but more circular shaped and confined to the upper hemisphere, i.e., less mirrored. Another less contrasted cool spot is seen on S2 centered at 0.83 


\section{S1}
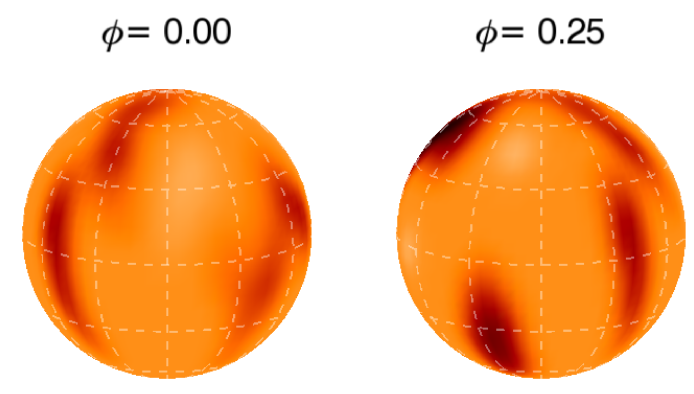

$\phi=0.25$

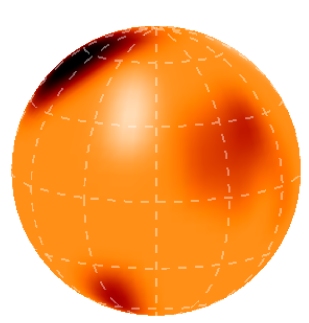

$\phi=0.00$

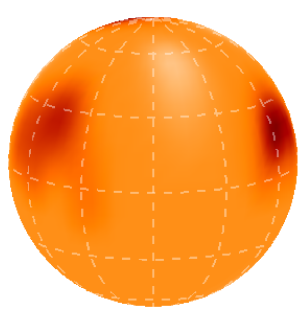

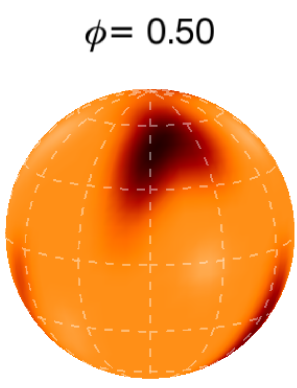
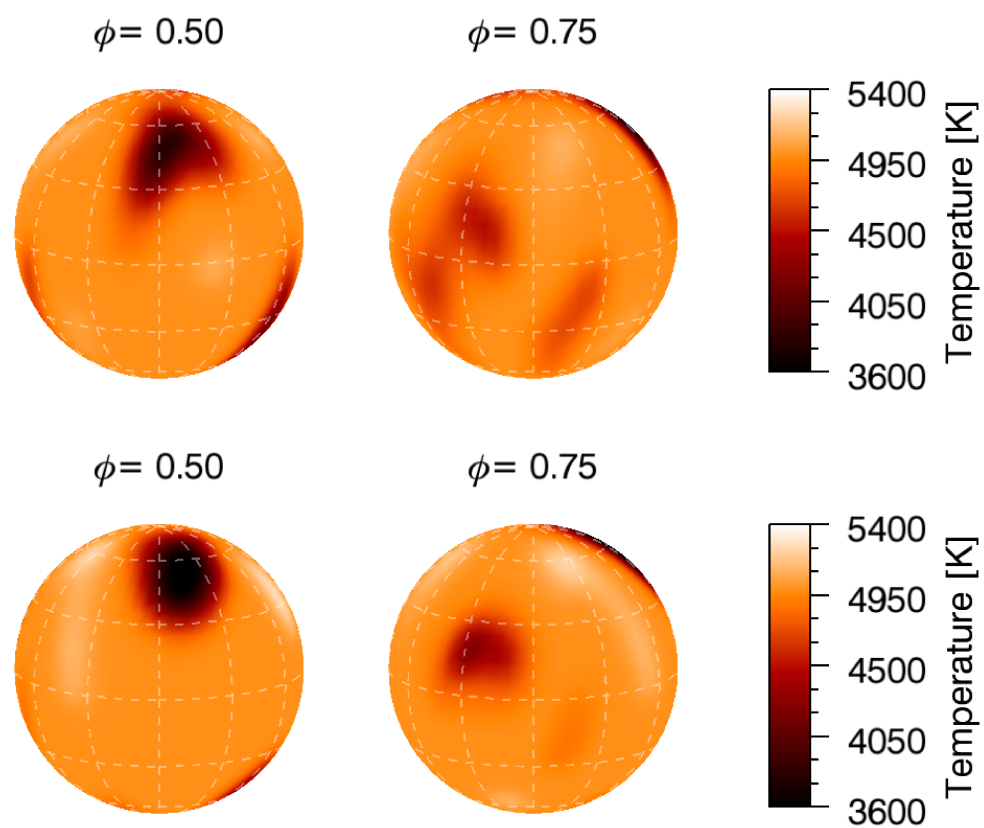

Fig. 6. Two Doppler images of V471 Tau from the 2005 data (S1 and S2). The surface temperature reconstructions are plotted in four rotational phases.
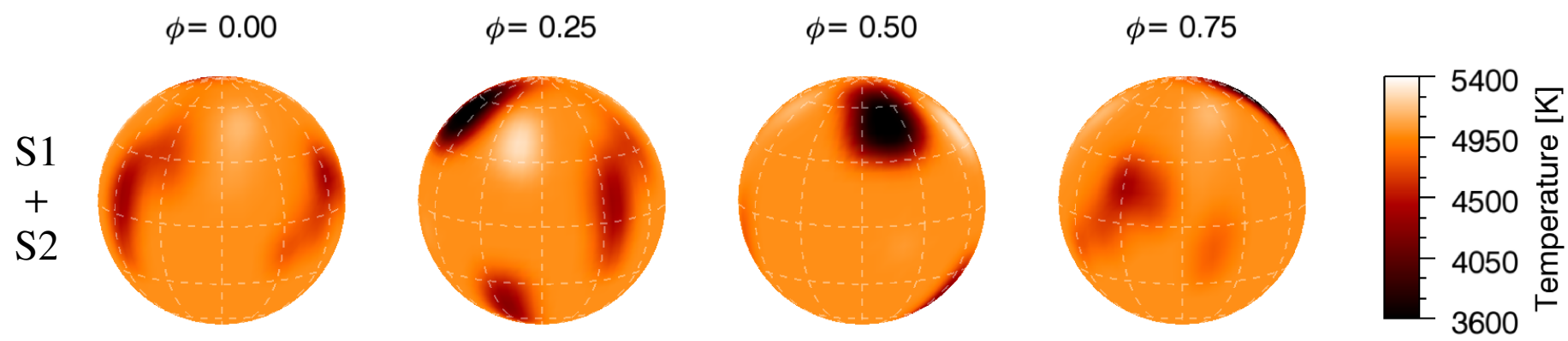

Fig. 7. Combined Doppler image of V471 Tau derived from all the available spectra from 2005 (S1+S2). The surface temperature map is plotted in four rotational phases.

phase and $20^{\circ}$ latitude, which, again, has a weaker but more extended precursor on S1.

Some weak bright features are also seen, but their contrast hardly reaches $\Delta T \approx+200 \mathrm{~K}$ and their location and shape are more changeful when comparing S1 image against S2. Their presence is even less salient on the average $(\mathrm{S} 1+\mathrm{S} 2)$ image except the one at 0.3 phase. This feature, however, raises reliability issues. We know that spurious bright features can appear as an artifact of Doppler imaging coming from insufficient phase coverage (e.g. Lindborg et al. 2014). The phase coverages for both S1 and S2 were fairly good, the only remarkable phase gaps were between 0.288-0.500 and 0.171-0.318 in S1 and S2 datasets, respectively. This may explain the bright spot at 0.3 phase on the $\mathrm{S} 2$ reconstruction and account for some minor inconsistencies between S1 and S2 around these phase gaps. All in all, we think that those bright spots are mostly artifacts.

\subsubsection{Doppler images from the $2014 / 15$ CFHT spectra}

Doppler images for the 2014/15 observing season (S3, S4) are presented in Fig. 8, for the corresponding line profile fits see
Figs. A.3 A.4 Again, the most dominant cool features in two subsequent maps appear at similar locations. However, neither the weaker cool features nor the bright features are particularly consistent. For example, bright features are more apparent in the $\mathrm{S} 3$ reconstruction, compared with $\mathrm{S} 4$. This is not surprising when keeping in mind that due to the unfavourable data distribution the selected spectra cover $\approx 20$ days for S3 and ten days for S4. Moreover, the time gap between the mean HJDs of S3 and $\mathrm{S} 4$ is $\approx 15$ days, therefore incoherences between the two images are more visible. The combined Doppler map derived from all of the spectra $(\mathrm{S} 3+\mathrm{S} 4)$ in Fig. 9 indicates that the most coherent and most striking feature is the high latitude $\left(\beta \approx 55^{\circ}\right)$ cool spot at around $\approx 0.4$ phase, recalling the $\mathrm{S} 1$ and $\mathrm{S} 2$ reconstructions. This cool spot may be coupled to the orbital phase, as suggested in Schrijver \& Zwaan (1991). We conclude that the weak and inconsistent features in the S3 and S4 reconstruction, being cool or bright, can either be the result of imperfections of the reconstruction or come from the unfavourable conditions of the data distribution. 

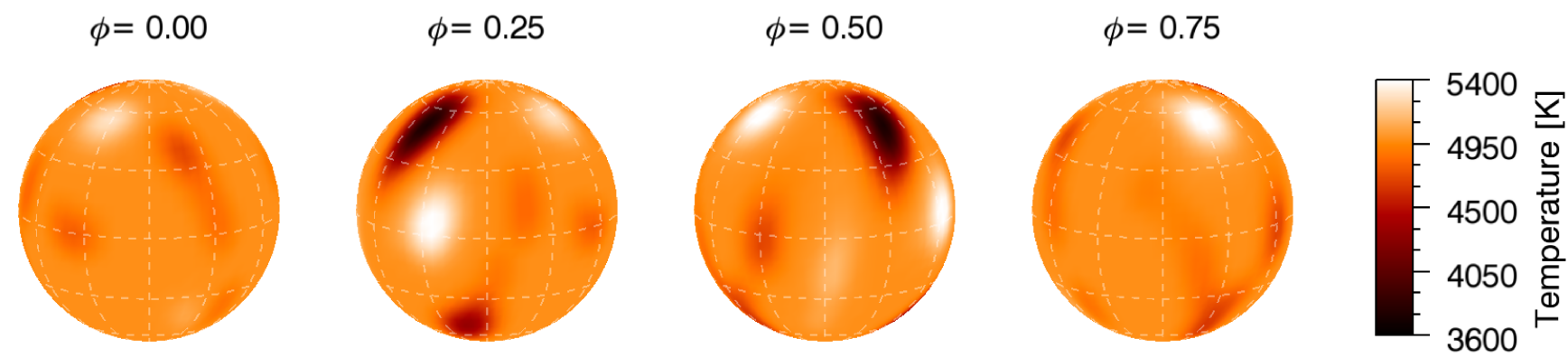

$\phi=0.00$

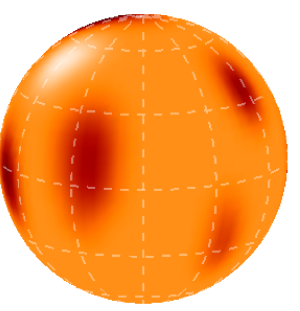

$\phi=0.25$

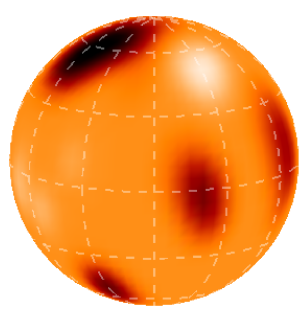

$$
\phi=0.50
$$

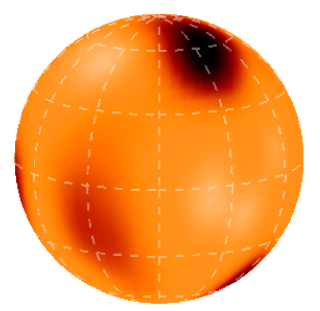

$\phi=0.75$

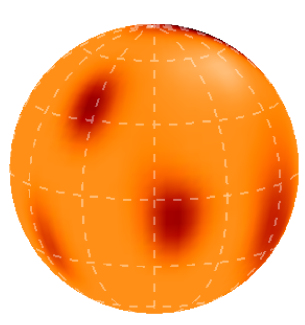

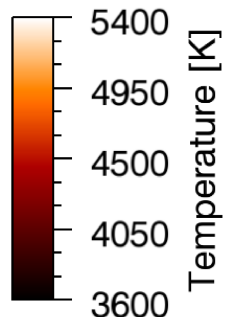

Fig. 8. Two Doppler images of V471 Tau for the second observing run at the turn 2014/2015 (S3 and S4). The surface temperature reconstructions are plotted in four rotational phases.
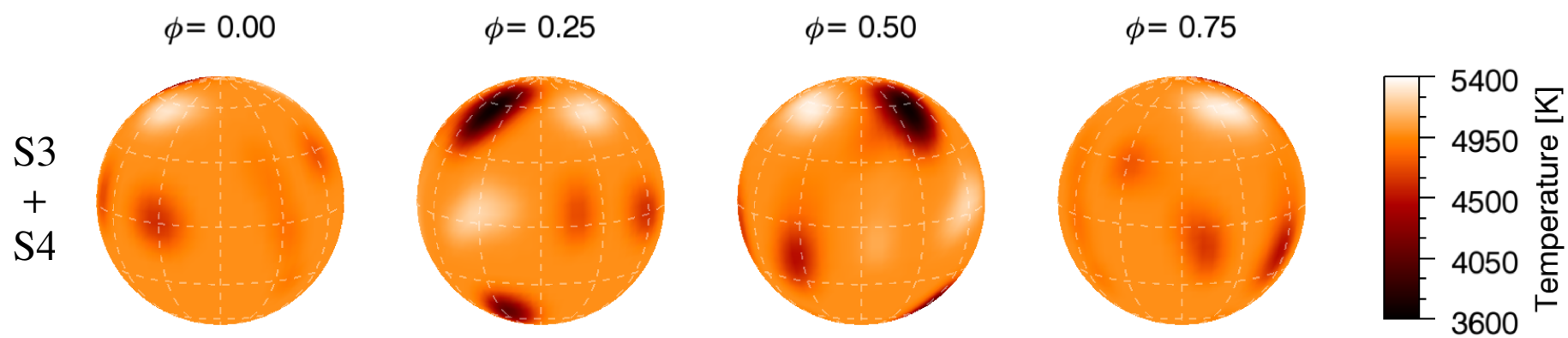

Fig. 9. Combined Doppler image of V471 Tau derived from all the spectra in the second observing run at the turn 2014/2015 (S3+S4). The surface temperature map is plotted in four rotational phases.

\subsection{Differential rotation}

Surface differential rotation can be measured by longitudinally cross-correlating consecutive Doppler images (Donati \& Collier Cameron 1997, Kóvári et al. 2004), and fitting the latitudinal correlation peaks in the resulting cross-correlation function (ccf) map by a quadratic rotational law:

$\Omega(\beta)=\Omega_{\mathrm{eq}}-\Delta \Omega \sin ^{2} \beta$,

where $\Omega(\beta)$ is the angular velocity at $\beta$ latitude, $\Omega_{\mathrm{eq}}$ is the angular velocity of the equator, and $\Delta \Omega=\Omega_{\mathrm{eq}}-\Omega_{\text {pole }}$ gives the difference between the equatorial and polar angular velocities. With these, the dimensionless surface shear parameter $\alpha_{\mathrm{DR}}$ is defined as $\alpha_{\mathrm{DR}}=\Delta \Omega / \Omega_{\mathrm{eq}}$, leading the following form:

$\Omega(\beta)=\Omega_{\mathrm{eq}}\left(1-\alpha_{\mathrm{DR}} \sin ^{2} \beta\right)$.

In our case two ccf maps can be composed, one for the two consecutive maps in the first observing season (S1, S2) and another one for the two maps in the second season (S3, S4). After the necessary normalization procedure the two ccf maps are combined to get an average ccf map (for the applied method

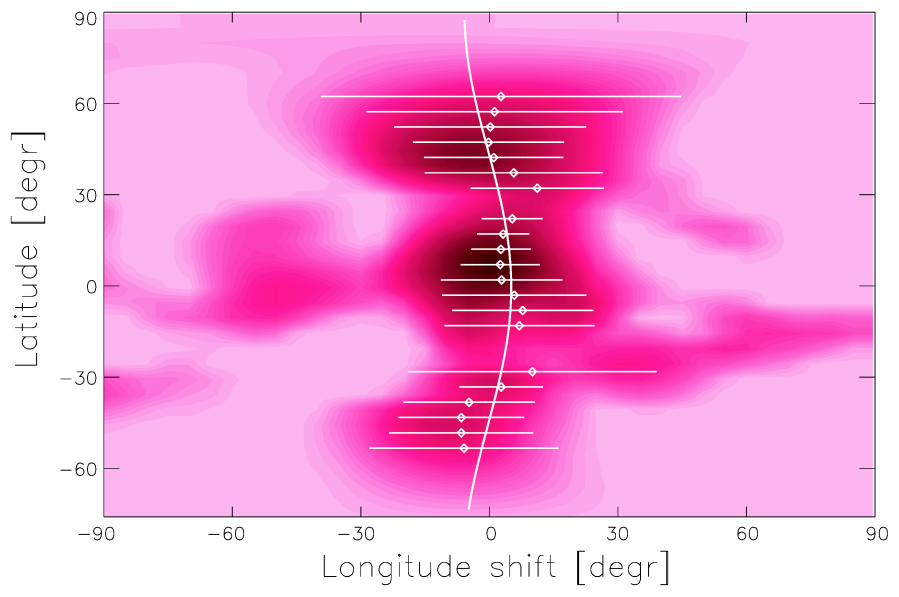

Fig. 10. Average cross-correlation function map derived from the subsequent Doppler images plotted in Fig.6 and Fig.8 Black represents strong correlation while light pink indicates no correlation. The quadratic sine fit (solid line) applied to the ridge of the correlation pattern (dots with error bars) suggests a solar-type rotational law with a very weak shear of $\alpha_{\mathrm{DR}}=0.0026 \pm 0.0006$. See Sect. 5.3 for details. 


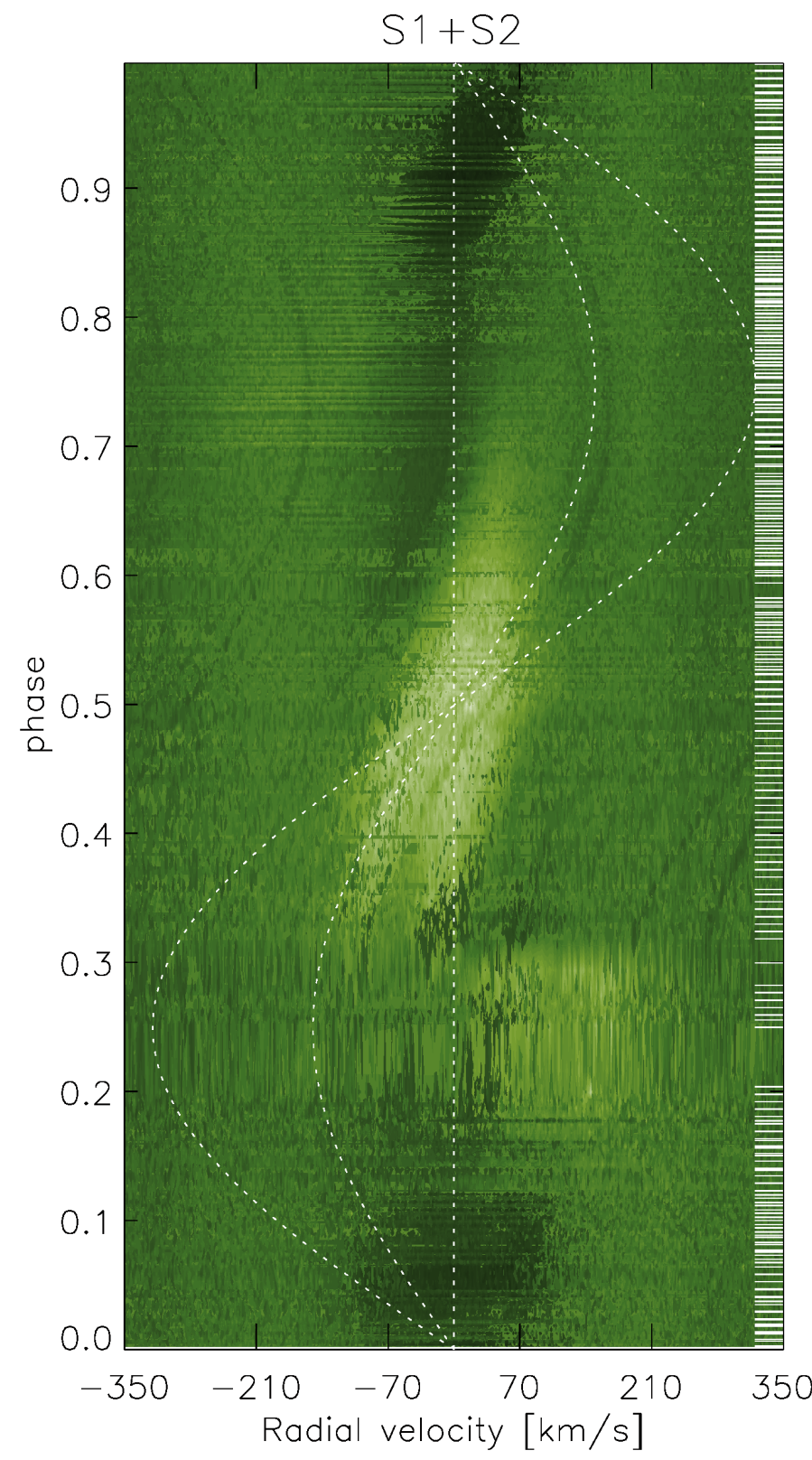

Fig. 11. Dynamic $\mathrm{H} \alpha$ spectrum from the 2005 data $(\mathrm{S} 1+\mathrm{S} 2)$. The individual 1-D $\mathrm{H} \alpha$ spectra along the orbital phase are plotted in the rest frame of the $\mathrm{K}$ star. Dark green corresponds to deep absorption, while light yellow indicates strong emission. The large amplitude $\left(\approx 320 \mathrm{~km} \mathrm{~s}^{-1}\right)$ sine curve marks the radial velocity of the white dwarf, the smaller amplitude $\left(\approx 150 \mathrm{~km} \mathrm{~s}^{-1}\right)$ sinusoid is the radial velocity of the centre of mass of the binary system. Tick marks on the right indicate the phases of the observations.

dubbed ACCORD see e.g., Kővári et al. 2012, 2015). The correlation pattern in the ultimate average ccf map (Fig.10) is fitted by a rotational law according to Eq.5. The resulting fit indicates a very weak solar-type differential rotation, with $\Omega_{\mathrm{eq}}=$ $691.57 \pm 0.13^{\circ} /$ day and $\alpha_{\mathrm{DR}}=0.0026 \pm 0.0006$ shear parameter, i.e., almost solid body rotation, in a fair agreement with the rough estimation from the $K 2$ photometry (see Sect.4.2). From the fitted $\Omega_{\mathrm{eq}}$ and $\alpha_{\mathrm{DR}}$ values we get $\beta_{\mathrm{cor}} \approx 43^{\circ}$ for the co-rotating latitude, i.e., where rotation is synchronized exactly to the orbit.

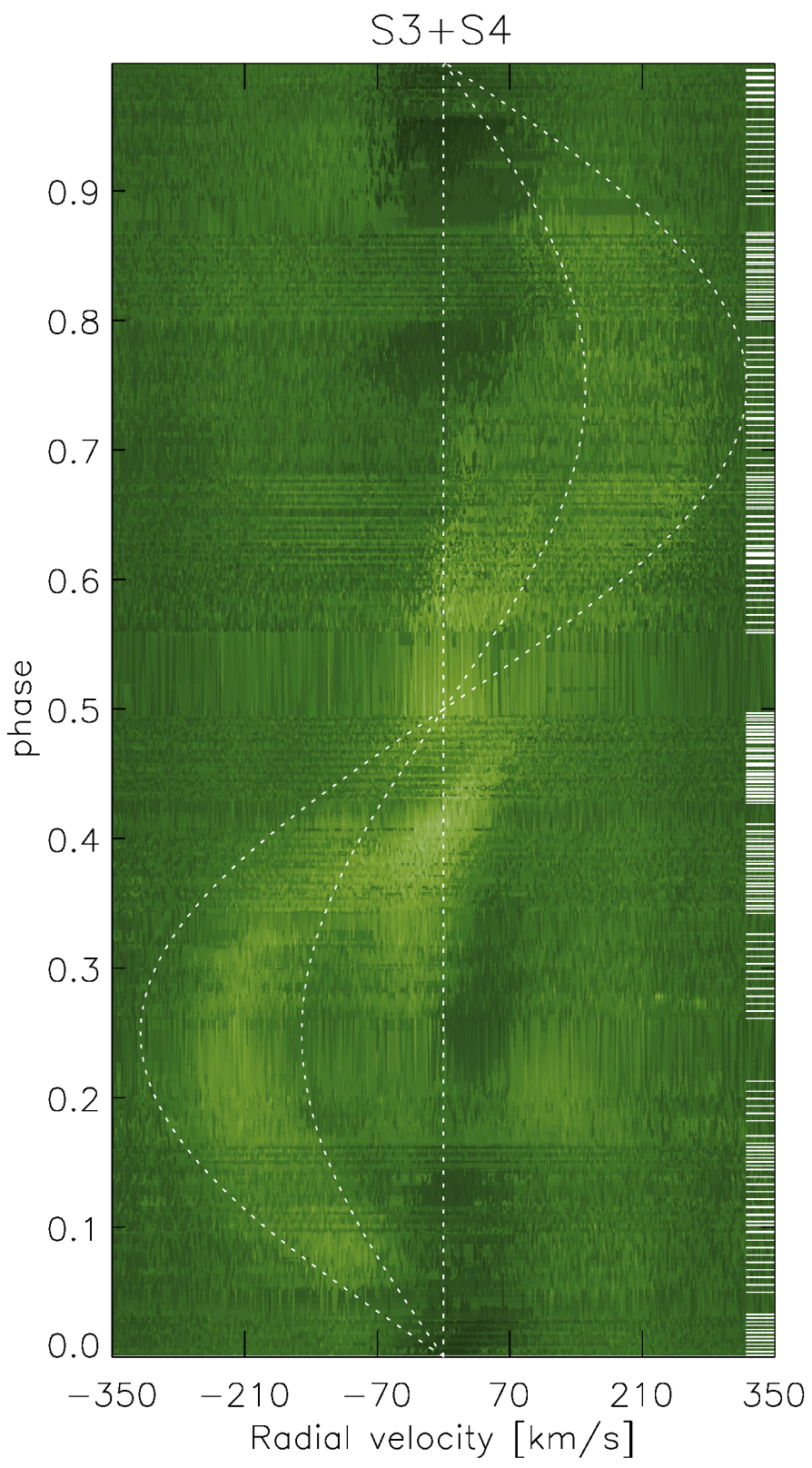

Fig. 12. Dynamic H $\alpha$ spectrum from the $2014 / 2015$ data (S3+S4). Otherwise as in Fig.11]

\section{Chromospheric and coronal activity}

\subsection{Variability of the $\mathrm{H} \alpha$ line profile}

$\mathrm{H} \alpha$ line profile variation is capable to unfold chromospheric activity and presumably associated mass motions like CMEs. From the available time series of spectra we create time-slice plots for both observing runs. The resulting dynamic spectra are shown in Figs. 11, 12 The plot for the first observing run $(\mathrm{S} 1+\mathrm{S} 2)$ indicates absorption around 0.0 phase when the white dwarf is near or in eclipse. On the other hand, a strong emission develops between around 0.35-0.6. Clearly, the peaks of the absorption and the emission profiles stay within the $\pm 91 \mathrm{~km} \mathrm{~s}^{-1}$ projected equatorial velocity range of the $\mathrm{K}$ star, suggesting that the sources are related to some surface features. Especially, the emission around the secondary eclipse may indicate relation with the dominant cool photospheric spot at around 0.45 phase (cf. Fig. 6). There may also be some extra emission on or over the receding side of 
the K star between 0.2-0.3 phase, however, this part of the data has the lowest quality and even the phase coverage is not satisfactory, therefore we cannot make a definite statement about this feature.

The $\mathrm{H} \alpha$ behaviour from the $2014 / 2015$ data $(\mathrm{S} 3+\mathrm{S} 4)$ in Fig. 12) shows similarities to the dynamic $\mathrm{H} \alpha$ spectrum from the 2005 data, but quite different features are also present. Similar features are the absorption centered around 0.0 phase and the emission between 0.35-0.65 phase, this latter less conspicuous but still around the phase of the most prominent cool spot (see Fig. 8). Being within the radial velocity range of the $\mathrm{K}$ dwarf, it is very likely that these features are localized on the surface of the red dwarf. However, in addition to the similarities there is a very different emitting phenomenon located between the centre of mass of the system and the white dwarf. This emission is stronger in the first half of the orbital phase, but still visible during the second half. This suggests the presence of a clump of emitting plasma corotating with the system in the vicinity of the inner Lagrangian (L1) point, as already reported by Young et al. (1991). We note finally, that there may be some extra emission on the receding side of the $\mathrm{K}$ star around $\phi=0.2$, recalling Fig. 11, but the phase coverage is not satisfactory around and the emission peaks are not so strong, therefore, again, we could only guess about its reliability, nature and possible origin.

For further discussion about the peculiar emission from beyond the L1 point see Sect.7.4.

\section{2. $X$-ray emission from the $K d$ warf}

The ROSAT pointed observations from 1991 were already analyzed by Wheatley (1998) who focused on the second, longer exposure that we also use here. The full wavelength coverage of the PSPC detector is $0.1-2.5 \mathrm{keV}$, i.e. the softer part is dominated by the emission from the white dwarf. Wheatley (1998) presented the light curve of the observation in a soft $(0.1-0.4 \mathrm{keV})$ and a hard (0.4-1.2 keV) band. The soft band clearly shows the (partly covered) eclipse, which is not seen in the hard band, confirming that the harder X-ray emission stems from the $\mathrm{K}$ dwarf only. Both bands show variability, but no large flares. The ROSAT data are, however, affected by many data gaps due to the spacecraft orbit. Therefore, we simply use the average flux from this observation as the quasi-quiescent state for this epoch. The $0.1-2.5 \mathrm{keV}$ flux from the $\mathrm{K}$ dwarf only (determined by spectral modelling in Wheatley (1998)) amounts to $(4.0 \pm 0.2) \times 10^{-12} \mathrm{erg} \mathrm{cm}^{-2} \mathrm{~s}^{-1}$.

In 1996, V471 Tau was observed by ASCA. This observation is described and analyzed in detail by Still \& Hussain (2003). Their light curve from the combined SIS and GIS data in the energy range $0.3-10 \mathrm{keV}$ shows some variability which may be due to small flare events, but the number of potentially flaring data points is small compared to the full light curve. Still \& Hussain (2003) used the whole observation for spectral modeling (due to the rather low count rate) and obtained an average flux of $2.4 \times 10^{-12} \mathrm{erg} \mathrm{cm}^{-2} \mathrm{~s}^{-1}$ valid for the $0.5-10 \mathrm{keV}$ range, which we adopt here as well.

One year later, V471 Tau was observed with the ROSAT High Resolution Imager (HRI). This instrument has an energy range of $0.1-2.5 \mathrm{keV}$, but due to its negligible energy resolution the data cannot be binned in energy to remove the contribution from the white dwarf. Therefore, we extracted the light curve of the whole observation using the xselect task from the HEASof ${ }^{1}$ package. This observation covered a part of one eclipse. There-

1 https://heasarc.gsfc.nasa.gov/docs/software/heasoft/ fore, we used xselect to extract an image for the time interval of the eclipse only (thus containing only emission from the $\mathrm{K}$ dwarf) and determined the count rate with the ximage/sosta task, which performs also background subtraction and corrects for vignetting, deadtime, and PSF. Although the full light curve did not show any large flares, the short duration of the usable time interval makes this data point more uncertain.

The Chandra observations from 2002 were already analyzed in several studies (Drake \& Sarna 2003; Ness et al. 2004; GarcíaAlvarez et al. 2005). The HRC-S/LETG instrument provides high-resolution spectroscopy in the $0.07-7.29 \mathrm{keV}$ range. The light curve shown by García-Alvarez et al. (2005) does not include any strong flares, so the average flux of the observation was adopted. This was taken from Ness et al. (2004) who extracted the flux in the smaller 5.15-38.19 $\stackrel{\AA}{ }$ wavelength band (quoted luminosity of $1.1234 \times 10^{30} \mathrm{erg} \mathrm{s}^{-1}$ ), i.e. with negligible contribution from the white dwarf.

The first XMM Newton observation from 2004 was analyzed using the XMM Newton Scientific Analysis System (SAS) Version 17.0.0. We only use data from the EPIC/PN detector, as the simultaneous EPIC/MOS and RGS data have lower S/N ratio. The SAS task epproc was used to create an event list from the raw odf file for the EPIC/PN detector. We extracted source and background regions in $d s 9$ and use the SAS tasks evselect to create the light curves and epiclccorr the perform background subtraction and all necessary corrections (vignetting, deadtime, etc.). We use the energy band $0.3-10 \mathrm{keV}$ to minimize potential contamination from the white dwarf at lower energies, and because we use this energy range for the common analysis of all instruments. This observation is affected by a large flare event previously analyzed by Pandey \& Singh (2008) and significant variability. Therefore, we needed to use a restricted time range that most likely resembles a quasi-quiescent state. We extract the last $18783 \mathrm{~s}$ of the observation which seems to be free of any trends, although there is still strong scatter in the light curve.

In 2015, V471 Tau was observed by Swift. We inspected the light curves from the XRT instrument created by the online tool of the Swift Science Center ${ }^{2}$ (Evans et al. 2007, 2009). There are no strong flares in the data, so we simply adopt the averaged count rate from all observations in the $0.3-10 \mathrm{keV}$ band.

The second XMM Newton observation from 2019 was processed an analyzed in the same way as described before. This observation was also affected by a large flare and strong variability, which required to extract the first $9381 \mathrm{~s}$ only, which seems to display a rather constant count rate without trends.

\subsection{Evolution of the $X$-ray luminosity}

To compare the X-ray emission from many different instruments requires to either convert the fluxes taken from the literature to a common energy range or to obtain the appropriate count-toflux conversion factors for the different instruments. We use the tool WebPIMMS ${ }^{3}$ to perform the necessary conversions. This requires input of the Galactic hydrogen absorption and a spectral model for the source.

To constrain the plasma parameters of the $\mathrm{K}$ dwarf in V471 Tau we perform spectral modeling of the two XMM Newton data sets and Swift. For XMM Newton, we extract source and background spectra of the selected quasi-quiescent time ranges using evselect and create the required rmf and arf files using

\footnotetext{
https://www.swift.ac.uk/user_objects/

3 https://heasarc.gstc.nasa.gov/cgi-bin/Tools/ w3pimms/w3pimms.pl
} 
Table 4. Spectral fits to the quiescent parts of the two XMM Newton observations from 2004 and 2019 and the Swift data from 2015. Errors refer to the $90 \%$ confidence intervals returned by Xspec. The last column gives the goodness-of-fit and the degrees of freedom.

\begin{tabular}{crrrrrrrr}
\hline Data & $\begin{array}{r}k T_{1} \\
{[\mathrm{eV}]}\end{array}$ & $\begin{array}{r}E M_{1} \\
{\left[10^{52} \mathrm{~cm}^{-3}\right]}\end{array}$ & $\begin{array}{r}k T_{2} \\
{[\mathrm{eV}]}\end{array}$ & $\begin{array}{r}E M_{2} \\
{\left[10^{52} \mathrm{~cm}^{-3}\right]}\end{array}$ & $\begin{array}{r}Z \\
{\left[Z_{\odot}\right]}\end{array}$ & $\begin{array}{r}T_{\text {av }} \\
{[\mathrm{MK}]}\end{array}$ & $\begin{array}{r}E M_{\text {tot }} \\
{\left[10^{52} \mathrm{~cm}^{-3}\right]}\end{array}$ & $\chi_{\text {red }}^{2}(\mathrm{DOF})$ \\
\hline \hline XMM 2004 & $0.34_{-0.02}^{+0.05}$ & $3.1_{-0.2}^{+0.2}$ & $0.96_{-0.02}^{+0.02}$ & $6.7_{-0.4}^{+0.4}$ & $0.18_{-0.02}^{+0.02}$ & 8.86 & 9.9 & $1.503(58)$ \\
XMM 2019 & $0.36_{-0.03}^{+0.04}$ & $2.6_{-0.3}^{+0.3}$ & $1.01_{-0.02}^{+0.02}$ & $6.0_{-0.4}^{+0.4}$ & $0.18_{-0.02}^{+0.02}$ & 9.48 & 8.6 & $1.043(58)$ \\
Swift 2015 & $1.03_{-0.08}^{+0.15}$ & $11.9_{-2.3}^{+2.5}$ & - & - & $0.13_{-0.05}^{+0.07}$ & - & - & $0.999^{a}(132)$ \\
\hline
\end{tabular}

Notes. ${ }^{(a)}$ Pearson $\chi_{\text {red }}^{2}$ due to use of Cash statistics for fitting.

rmfgen and arfgen. We use specgroup to group the files and select a minimum of 20 counts per bin and not oversampling the intrinsic energy resolution by more than a factor of three. We fit the spectra using Xspec $4^{4}$ with a model including Galactic absorption and a sum of APEC (Smith et al. 2001) plasma models. As the Galactic absorption is not very well constrained by a fit to energy ranges $>0.3 \mathrm{keV}$ we fix the $\mathrm{H}$ column density towards V471 Tau to its measured value of $N_{\mathrm{H}}=1.62 \times 10^{18} \mathrm{~cm}^{-2}$ (Wood et al. 2005). This has a negligible effect on the results, as this value is low because the star is nearby. For the fitting process, we restrict the energy range further to $0.3-3.9 \mathrm{keV}$ due to low signal at higher energies. At both epochs, we find best fits with two APEC plasma components with the same coronal abundances (Table 4). The results are broadly consistent with each other, as well as other spectral analyses in the literature. We also tried to fit the Swift spectrum obtained from the online tool (Evans et al. 2009), but due to the small number of counts it was not possible to fit a 2-T plasma model. An acceptable fit was obtained for a 1-T model using Cash statistics (Cash|1979), yielding a temperature comparable to the hotter component of the more reliable XMM Newton fits and an emission measure similar to the total one obtained for XMM Newton. All fits predict subsolar coronal abundances $\left(Z \sim 0.2 Z_{\odot}\right)$, consistent with previous studies. Table 4 summarizes the plasma parameters obtained from the Xspec fits, together with the calculated average emission measure-weighted temperature $T_{\mathrm{av}}$ and total emission measure $E M_{\text {tot }}$ for the 2-T fits.

As we do not find significant differences of coronal plasma parameters from the analyzed observations, we adopt a common parameter set for all flux conversions. For simplicity, we use a 1-T APEC model in WebPIMMS with a mean temperature of $8.9 \mathrm{MK}(\log T=6.95)$. We fix the Galactic $N_{\mathrm{H}}$ absorption to the value from Wood et al. (2005) and the coronal metal abundance to $0.2 Z_{\odot}$. Using a 2-T model with parameters similar to those obtained in the XMM-Newton fits does not change the results significantly. To estimate uncertainties for the conversion factors, we run WebPIMMS with $\log T=6.9$ and $\log T=7.0$, corresponding to an uncertainty of the mean temperature by about $10 \%$, which is slightly larger than what we calculate from the XMM-Newton spectra $(3-4 \%)$. This results in uncertainties on the conversion factors by $\lesssim 3 \%$.

After converting the fluxes taken from the literature and the count rates of the specific instruments to a common energy range of $0.3-10 \mathrm{keV}$, we use the distance from Table 2 to convert the fluxes to luminosities. The results are shown in Fig. 13 . The displayed uncertainties on the X-ray luminosities include the statistical uncertainties of the flux/count rate measurements, the flux conversion factors, and the distance. The X-ray luminosity of the $\mathrm{K}$ dwarf shows variations over the years, with the maximum

\footnotetext{
4 https://heasarc.gsfc.nasa.gov/xanadu/xspec/
}

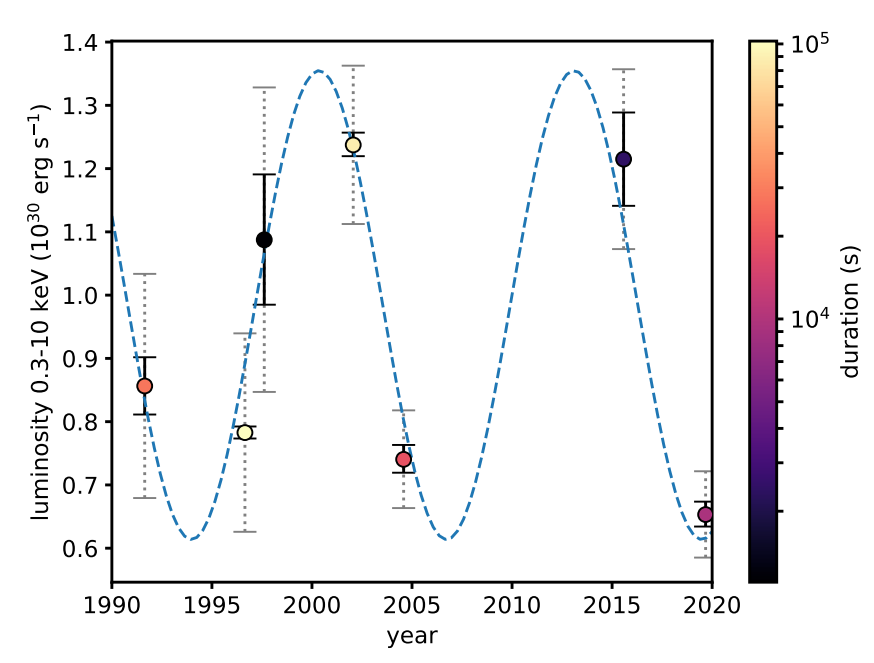

Fig. 13. Evolution of the X-ray luminosity of the K dwarf in V471 Tau from the observations summarized in Table1. The duration of the selected quiescent ranges is color coded. Solid error bars denote flux errors (see text), dotted bars additionally the estimated cross-calibration uncertainty. The blue dashed line indicates the best fit with a sinusoidal function with a period of $\sim 12.7 \mathrm{yr}$.

data point being $90 \%$ higher than the minimum one. As there are only seven data sets spanning almost 30 years, some with exposure times for the quasi-quiescent time intervals as short as $\sim 20 \mathrm{~min}$, a detailed investigation of a possible cycle is not feasible. Moreover, the data stem from a variety of different instruments and cross-calibration issues are expected to be present (see the detailed discussion in Wargelin et al.|2017). To account for such instrument cross-calibration uncertainties, we add $10 \%$ for Chandra, XMM-Newton and Swift data, as well as $20 \%$ for the older ROSAT and ASCA data to the luminosity uncertainties (Snowden 2002, Tsujimoto et al.|2011).

Despite the limitations of the available data, we fitted a sinusoidal function to the X-ray luminosities in Fig.13. The blue dashed line in the plot corresponds to a period of about $12.7 \mathrm{yr}$, which can be interpreted as activity cycle. We stress again, however, that the uneven spacing of the observations, the small number of data points, as well as uncertainties of instrumental crosscalibration, do not allow a definitive conclusion about the existence of an X-ray cycle and its properties.

\section{Discussions}

\subsection{Weak differential rotation and active longitude}

From our Doppler reconstructions we have measured a very weak solar-type surface differential rotation for the $\mathrm{K}$ star 
(Sect.5.3), Such a weak shear, however, is not surprising, as we essentially expect something similar for fast rotating latetype dwarf stars (see the empirical study by Kővári et al. 2017, and their references). In addition, synchronization due to tidal dissipation in close binaries may suppress the rotational shear (Scharlemann 1982). In Hussain et al. (2006) authors reported almost rigid body rotation for V471 Tau with $\alpha_{\mathrm{DR}}$ of $0.0001 \pm 0.0005$ shear coefficient. In comparison, our result of $\alpha_{\mathrm{DR}}=0.0026 \pm 0.0006$ is still weak, but an order of a magnitude stronger shear based on using much more data and our well-established cross-correlation technique ACCORD (for some applications and tests see, e.g. Kővári et al. 2004, 2012, 2014. 2015, and their references). This technique amplifies independent cross-correlation signals from different epochs to multiply the credibility and reliability of the result. We note finally, that our larger shear coefficient is also supported by the short term photometric period changes (see Sect.4.2).

The Doppler reconstructions performed in our study indicate that the most dominant surface spot located around 0.400.45 phase at $\beta \approx 50-55^{\circ}$ latitude may be a permanent feature (see Figs. 6 8). In the Doppler imaging study by Ramseyer et al. (1995) authors have found that a dominant cool spot appeared at the longitude facing the white dwarf in all their four Doppler maps (1992 September, October, December and 1993 December). Moreover, the Doppler image from 2002 November (Hussain et al. 2006) has also indicated that the most prominent spot was directed toward the white dwarf.

Asymmetric tidal distortion may significantly alter internal dynamos of rapidly rotating convective stars in close binaries (Rottler et al. 2002), which can result in an active longitude around the sub-white dwarf point in the $\mathrm{K}$ dwarf component. Such a non-axisymmetric nature of spot activity is not unusual in close binary systems (Oláh 2006). However, it has also been shown that the condition for the excitation of stable, nonaxisymmetric fields is weak differential rotation (Scharlemann 1982; Ruediger \& Elstner 1994), which we firmly confirmed in the case of V471 Tau.

\subsection{Effective temperature rise around $\phi=0.5$ phase}

The $\approx 100 \mathrm{~K}$ variation of the surface temperature of the $\mathrm{K}$ dwarf along the orbital phase (see Fig. 3) may be interpreted as the irradiation effect of the white dwarf. In fact, we have no means to calculate what fraction of the radiative energy from the white dwarf is converted to thermal reprocessing in the photosphere of the K star. However, according to Young et al. (1988, their Table 2) the estimated ratio of the total luminous energy intercepted from the white dwarf to the bolometric output of the $\mathrm{K}$ star is $\approx 0.6 \%$, i.e., below the detection limit of $3 \%$ assumed for such a heating mechanism to be efficient in the photosphere. A similar conclusion has been drawn by O'Brien et al. (2001), who estimated that the luminosity of the $\mathrm{K}$ star would increase only by $1 \%$ due to the irradiation from the white dwarf, which would explain only $\approx 12 \mathrm{~K}$ temperature rise of the sub-white dwarf hemisphere. In contrast, the effective temperature change of $\approx 100 \mathrm{~K}$ in Fig. 3 would assume $\approx 8 \%$ luminosity difference. At this point we speculate that the photospheric temperature rise around $\phi=0.5$ may rather be associated with increased magnetic activity at the sub-white dwarf hemisphere of the $\mathrm{K}$ star. It has been learned that faculae-dominated active stars tend to become brighter when their magnetic activity level increases (Radick et al. 1990). We believe that the hemispheric difference in magnetic activity observed in V471 Tau can have a significant effect on the overall hemispheric temperature difference as well

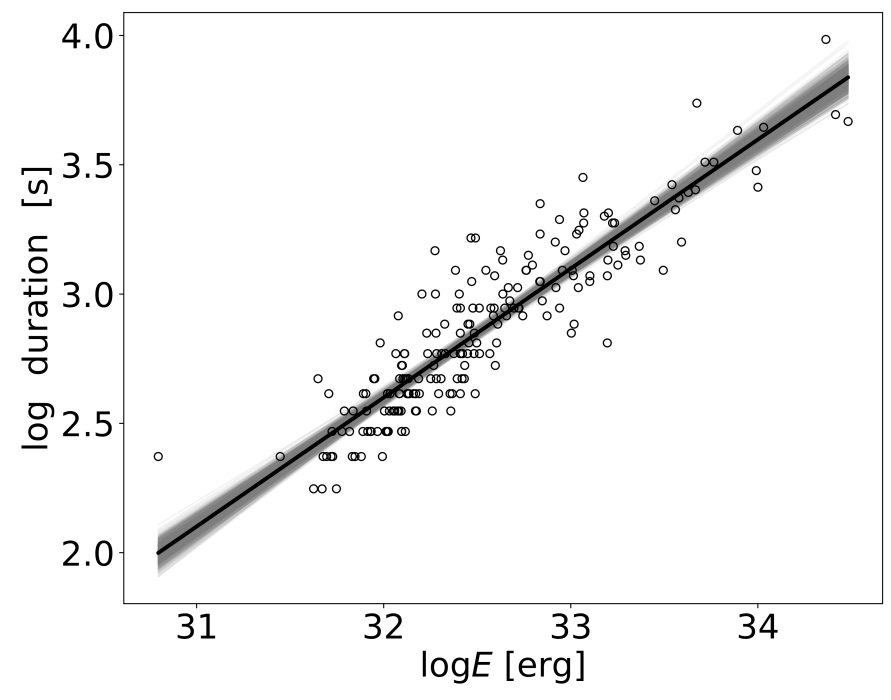

Fig. 14. Flare duration vs. flare energy from the $K 2$ data of V471 Tau. The best fit indicates a power-law relationship with $0.5 \pm 0.02$ index.

(cf. e.g. Gondoin 2008; Shapiro et al.2016). That is, small-scale photospheric bright faculae, permanently present around the active longitude facing the white dwarf, may infer such a rise in the effective temperature.

\subsection{Flare statistics}

We find that the 198 flares detected in the 71-day long $K 2$ data of V471 Tau are distributed randomly over the rotational phase, i.e. no significant phase dependency was found in the flare occurrence. The derived flare energies in Sect.4.3 range over four magnitudes. Recently, Kővári et al. (2020) have concluded that, in principle, differences between flare energies are due to size effect, regardless of energy range or spectral type (cf. Balona 2015). Accordingly, flare duration is supposed to increase with flare energy. In Fig. 14 we plot log $\Delta t$ flare duration as a function of $\log E_{\mathrm{f}}$ flare energy. The relationship on the $\log -\log$ plot fitted by a linear function yields a $0.50 \pm 0.02$ slope. In comparison, Maehara et al. (2015) suggested a generalized function between flare duration and flare energy with a power-law index (slope) of $\approx 1 / 3$.

It has been suggested (see e.g. Parker 1988, Hudson 1991, Saar \& Bookbinder 1998) that the more numerous small flaring events have an important role in heating the plasma in the transition region and the corona. This is supported by the power-law index of $\alpha \gtrsim 2.0$ derived from flare statistics of magnetically active $\mathrm{M}$ dwarf stars (e.g., Ilin et al. 2019, etc.). In comparison, $\alpha=1.7$ was obtained for 27 young K 5-7 stars in the Orion Nebula Cluster (Wolk et al. 2005). However, no such a difference was found from the flare statistics for $548 \mathrm{M}$ and $343 \mathrm{~K}$ dwarf stars ( $\alpha=1.82$ and 1.86, respectively) in Lin et al. (2019). Nevertheless, the $\alpha$ index of 1.8 obtained for V471 Tau (see Fig. 5) suggests that the dissipating energy of the flares should have a significant contribution in heating the upper atmosphere.

\subsection{Inter-binary $\mathrm{H} \alpha$ emission during activity maximum}

The cycle period of the sinusoid fitted to the X-ray luminosity variation in Fig. 13 is comparable to the $13 \mathrm{yr}$ period identified in timing residuals by Vaccaro et al. (2015) and a possible $\approx 10 \mathrm{yr}$ periodicity from an activity cycle mentioned by Kamiński 
et al. (2007). Moreover, Kamiński et al. (2007) stated that during observations with MOST in Dec 2005 the star was much less spotted than in the previous observations of Hussain et al. (2006) (from Nov 2002) and had only little photometric variability, which the authors interpreted as the evidence that the $\mathrm{K}$ dwarf was close to an activity minimum. This is consistent with our fit to the X-ray data.

According to Fig. 13, about four months ahead of the epochs of the S1-S2 Doppler reconstructions, in 2004 the X-ray luminosity of V471 Tau was around its minimum. While about six months after the epochs of the S3-S4 Doppler images, in 2015 the X-ray luminosity was much higher, according to the data point from Swift. Oddly, such a large difference is not seen when comparing the overall spottedness in the S1-S2 images with that of S3-S4, which are quite similar, despite the $\approx 9$-year time gap between them. However, the $\mathrm{H} \alpha$ line profile variability (see Figs. 11 12 showed indeed different behaviour in the two observing runs. The extra $\mathrm{H} \alpha$ emission from the inter-binary space (see Fig.12) might have something to do with the enhanced Xray luminosity in 2015 , i.e., around activity maximum. In deed, this is supported by the result in Young et al. (1991, see their Fig. 3) who also found external $\mathrm{H} \alpha$ emission component, originated from somewhere in the inter-binary space beyond the centre of mass of the system, expanding towards the white dwarf, definitely reminiscent of our result plotted in Fig.12 Moreover, their observations were obtained in Nov 3-4, 1988 and Jan 2930,1989 , i.e., close to the suspected maximum of the activity cycle ended in 1994 (according to our Fig.13). As a further support see also Rottler et al. (2002) for the secular $\mathrm{H} \alpha$ variability in 1985,1990 and 1992.

We expect that during the magnetic cycle, around activity maximum more and/or more extended magnetic loops develop high above the surface, reaching one stellar radius (Guinan et al. 1986), and this way more cool material enters the upper atmosphere in trapped clumps, similar to solar prominences. Large prominence-like condensations of cool material were reported from $\mathrm{H} \alpha$ observations of AB Dor Collier Cameron \& Robinson (1989), a single K0 dwarf, rotating as rapidly as V471 Tau. Normally, such a clump of cool material appears in absorption in $\mathrm{H} \alpha$, exactly what we actually see on the far side of the K star around zero phase. However, it can get into emission when heated due to the UV radiation from the white dwarf. The physical process behind heating proposed first by Young et al. (1988) involves fluorescence-induced $\mathrm{H} \alpha$ emission (see also Bois et al. 1991). This scenario could explain the $\mathrm{H} \alpha$ line variability both around the activity minimum (Fig.11) when there are no extended magnetic loops, and around maximum (Fig. 12) when extended loops with cool material trapped in them are present. Still, we caution that our ground-based spectroscopic data and the space observations did not overlap exactly and the available space data is limited. Therefore our conclusion about such a link between the peculiar $\mathrm{H} \alpha$ emission from the vicinity of the L1 point and the proximity of the activity cycle maximum may raise some criticism.

\section{Conclusions}

We confirm that the magnetic activity of the $\mathrm{K}$ dwarf component in V471 Tau is strongly influenced by the close white dwarf companion. Our related concluding remarks are as follows:

- We confirm that there is a permanent active longitude on the $\mathrm{K}$ star facing the white dwarf over decades, regardless of the actual phase of the magnetic cycle.
- We confirm a weak solar-type surface differential rotation on the surface of the $\mathrm{K}$ star, supporting theoretical expectations.

- We suggest that frequent flaring should have a significant contribution in heating the corona of the $\mathrm{K}$ star.

- From the long-term evolution of X-ray luminosity we confirm a magnetic activity cycle of $\approx 12.7 \mathrm{ys}$, whose traces appear also in the chromospheric $(\mathrm{H} \alpha)$ activity pattern.

- We find that the inter-binary $\mathrm{H} \alpha$ emission from the vicinity of the L1 point is correlated with the activity cycle: it intensifies during activity maximum and fades away during minimum.

Acknowledgements. This publication makes use of VOSA, developed under the Spanish Virtual Observatory project supported by the Spanish MINECO through grant AyA2017-84089. VOSA has been partially updated by using funding from the European Union's Horizon 2020 Research and Innovation Programme, under Grant Agreement No. 776403 (EXOPLANETS-A). This work was supported by the Hungarian National Research, Development and Innovation Office grant OTKA K131508, KH-130526, by the Lendület Program of the Hungarian Academy of Sciences, project No. LP2018-7/2019 and by the NKFIH grant 2019-2.1.11-TÉT-2019-00056. Authors acknowledge the financial support of the Austrian-Hungarian Action Foundation (98öu5, 1010̈u13). TB acknowledges the financial support of the Hungarian National Research, Development and Innovation Office grant NKFIH KH-130372. LK acknowledges the financial support of the Hungarian National Research, Development and Innovation Office grant NKFIH PD-134784.

\section{References}

Adibekyan, V. Z., Delgado Mena, E., Sousa, S. G., et al. 2012, A\&A, 547, A36 Balona, L. A. 2015, MNRAS, 447, 2714

Barstow, M. A., Schmitt, J. H. M. M., Clemens, J. C., et al. 1992, MNRAS, 255, 369

Bayo, A., Rodrigo, C., Barrado Y Navascués, D., et al. 2008, A\&A, 492, 277 Bois, B., Lanning, H. H., \& Mochnacki, S. W. 1991, AJ, 102, 2079

Bond, H. E., Mullan, D. J., O’Brien, M. S., \& Sion, E. M. 2001, ApJ, 560, 919

Borkovits, T., Rappaport, S. A., Hajdu, T., et al. 2020, MNRAS, 493, 5005

Carroll, T. A., Kopf, M., \& Strassmeier, K. G. 2008, A\&A, 488, 781

Carroll, T. A., Strassmeier, K. G., Rice, J. B., \& Künstler, A. 2012, A\&A, 548, A95

Cash, W. 1979, ApJ, 228, 939

Castelli, F. \& Kurucz, R. L. 2004, ArXiv Astrophysics e-prints [astro-ph/0405087]

Collier Cameron, A. \& Robinson, R. D. 1989, MNRAS, 238, 657

Csubry, Z. \& Kolláth, Z. 2004, in ESA Special Publication, Vol. 559, SOHO 14 Helio- and Asteroseismology: Towards a Golden Future, ed. D. Danesy, 396 Cully, S. L., Dupuis, J., Rodriguez-Bell, T., et al. 1996, in IAU Colloq. 152: Astrophysics in the Extreme Ultraviolet, ed. S. Bowyer \& R. F. Malina, 349 Donati, J.-F. \& Collier Cameron, A. 1997, MNRAS, 291, 1

Drake, J. J. \& Sarna, M. J. 2003, ApJ, 594, L55

Evans, P. A., Beardmore, A. P., Page, K. L., et al. 2009, MNRAS, 397, 1177

Evans, P. A., Beardmore, A. P., Page, K. L., et al. 2007, A\&A, 469, 379

Evren, S., Ibanoglu, C., Tunca, Z., \& Tumer, O. 1986, Ap\&SS, 120, 97

Flower, P. J. 1996, ApJ, 469, 355

Gaia Collaboration, Brown, A. G. A., Vallenari, A., et al. 2018, A\&A, 616, A1 García-Alvarez, D., Drake, J. J., Lin, L., Kashyap, V. L., \& Ball, B. 2005, ApJ, 621,1009

Gershberg, R. E. 1972, Ap\&SS, 19, 75

Gondoin, P. 2008, A\&A, 478, 883

Gossage, S., Conroy, C., Dotter, A., et al. 2018, ApJ, 863, 67

Guinan, E. F. \& Ribas, I. 2001, ApJ, 546, L43

Guinan, E. F. \& Sion, E. M. 1984, AJ, 89, 1252

Guinan, E. F., Wacker, S. W., Baliunas, S. L., Loesser, J. G., \& Raymond, J. C. 1986, in ESA Special Publication, Vol. 263, New Insights in Astrophysics. Eight Years of UV Astronomy with IUE, ed. E. J. Rolfe \& R. Wilson, 197200

Gustafsson, B., Edvardsson, B., Eriksson, K., et al. 2008, A\&A, 486, 951

Hardy, A., Schreiber, M. R., Parsons, S. G., et al. 2015, ApJ, 800, L24

Hawley, S. L., Davenport, J. R. A., Kowalski, A. F., et al. 2014, ApJ, 797, 121

Hill, C. A., Watson, C. A., Shahbaz, T., Steeghs, D., \& Dhillon, V. S. 2014, MNRAS, 444, 192

Howell, S. B., Sobeck, C., Haas, M., et al. 2014, PASP, 126, 398

Hudson, H. S. 1991, Sol. Phys., 133, 357

Hussain, G. A. J., Allende Prieto, C., Saar, S. H., \& Still, M. 2006, MNRAS, 367, 1699 
İbanoğlu, C., Evren, S., Taș, G., \& Çakırlı, Ö. 2005, MNRAS, 360, 1077

Ilin, E., Schmidt, S. J., Davenport, J. R. A., \& Strassmeier, K. G. 2019, A\&A, 622, A133

Jensen, K. A., Swank, J. H., Petre, R., et al. 1986, ApJ, 309, L27

Kamiński, K. Z., Ruciński, S. M., Matthews, J. M., et al. 2007, AJ, 134, 1206

Kővári, Zs., Bartus, J., Kriskovics, L., Vida, K., \& Oláh, K. 2014, in Magnetic Fields throughout Stellar Evolution, ed. P. Petit, M. Jardine, \& H. C. Spruit, Vol. 302, 198-199

Kővári, Zs., Korhonen, H., Kriskovics, L., et al. 2012, A\&A, 539, A50

Kővári, Zs., Kriskovics, L., Künstler, A., et al. 2015, A\&A, 573, A98

Kővári, Zs., Oláh, K., Günther, M. N., et al. 2020, A\&A, 641, A83

Kővári, Zs., Oláh, K., Kriskovics, L., et al. 2017, Astronomische Nachrichten, 338, 903

Kôvári, Zs., Strassmeier, K. G., Granzer, T., et al. 2004, A\&A, 417, 1047

Kriskovics, L., Kővári, Zs., Vida, K., et al. 2019, A\&A, 627, A52

Kupka, F., Piskunov, N., Ryabchikova, T. A., Stempels, H. C., \& Weiss, W. W. 1999, A\&AS, 138, 119

Lanza, A. F. 2020, MNRAS, 491, 1820

Lin, C. L., Ip, W. H., Hou, W. C., Huang, L. C., \& Chang, H. Y. 2019, ApJ, 873, 97

Lindborg, M., Hackman, T., Mantere, M. J., et al. 2014, A\&A, 562, A139

Maehara, H., Shibayama, T., Notsu, Y., et al. 2015, Earth, Planets, and Space, 67,59

Ness, J. U., Güdel, M., Schmitt, J. H. M. M., Audard, M., \& Telleschi, A. 2004, A\&A, 427, 667

Notsu, Y., Maehara, H., Honda, S., et al. 2019, ApJ, 876, 58

O’Brien, M. S., Bond, H. E., \& Sion, E. M. 2001, ApJ, 563, 971

Oláh, K. 2006, Ap\&SS, 304, 145

Pandey, J. C. \& Singh, K. P. 2008, MNRAS, 387, 1627

Parker, E. N. 1988, ApJ, 330, 474

Piskunov, N. \& Valenti, J. A. 2017, A\&A, 597, A16

Radick, R. R., Lockwood, G. W., \& Baliunas, S. L. 1990, Science, 247, 39

Ramseyer, T. F., Hatzes, A. P., \& Jablonski, F. 1995, AJ, 110, 1364

Rottler, L., Batalha, C., Young, A., \& Vogt, S. 2002, A\&A, 392, 535

Rucinski, S. M. 1981, Acta Astron., 31, 37

Ruediger, G. \& Elstner, D. 1994, A\&A, 281, 46

Saar, S. H. \& Bookbinder, J. A. 1998, in Astronomical Society of the Pacific Conference Series, Vol. 154, Cool Stars, Stellar Systems, and the Sun, ed. R. A. Donahue \& J. A. Bookbinder, 1560

Scharlemann, E. T. 1982, ApJ, 253, 298

Schrijver, C. J. \& Zwaan, C. 1991, A\&A, 251, 183

Shapiro, A. I., Solanki, S. K., Krivova, N. A., Yeo, K. L., \& Schmutz, W. K. 2016, A\&A, 589, A46

Smith, R. K., Brickhouse, N. S., Liedahl, D. A., \& Raymond, J. C. 2001, ApJ, 556, L91

Snowden, S. L. 2002, arXiv e-prints, astro

Still, M. \& Hussain, G. 2003, ApJ, 597, 1059

Taylor, B. J. 2006, AJ, 132, 2453

Tsujimoto, M., Guainazzi, M., Plucinsky, P. P., et al. 2011, A\&A, 525, A25

Vaccaro, T. R., Wilson, R. E., Van Hamme, W., \& Terrell, D. 2015, ApJ, 810, 157

Valenti, J. A. \& Fischer, D. A. 2005, ApJS, 159, 141

Vida, K. \& Roettenbacher, R. M. 2018, A\&A, 616, A163

Wargelin, B. J., Saar, S. H., Pojmański, G., Drake, J. J., \& Kashyap, V. L. 2017, MNRAS, 464, 3281

Wheatley, P. J. 1998, MNRAS, 297, 1145

Wilson, R. E. 1953, Carnegie Institute Washington D.C. Publication, 0

Wolk, S. J., Harnden, F. R., J., Flaccomio, E., et al. 2005, ApJS, 160, 423

Wood, B. E., Redfield, S., Linsky, J. L., Müller, H.-R., \& Zank, G. P. 2005, ApJS, 159,118

Young, A., Klimke, A., Africano, J. L., et al. 1983, ApJ, 267, 655

Young, A., Rottler, L., \& Skumanich, A. 1991, ApJ, 378, L25

Young, A., Skumanich, A., \& Paylor, V. 1988, ApJ, 334, 397 


\section{Appendix A: Log of spectroscopic data}

Table A.1. List of ESPaDOnS spectra of V471 Tau taken from the CFHT Science Archive. Given are the Heliocentric Julian Dates (HJD-2 450000 ), dates (dd.mm.yyyy), rotational phases computed using Eq. 1 signal-to-noise ratios $(\mathrm{S} / \mathrm{N})$ for the four subsets $(\mathrm{S} 1-\mathrm{S} 4)$ formed for Doppler imaging.

\begin{tabular}{|c|c|c|c|c|}
\hline HJD & Date & Phase & $\mathrm{S} / \mathrm{N}$ & Subset \\
\hline 3718.714 & 14.12 .2005 & 0.703 & 60 & $\mathrm{~S} 1$ \\
\hline 3718.717 & 14.12 .2005 & 0.709 & 58 & $\mathrm{~S} 1$ \\
\hline 3718.720 & 14.12 .2005 & 0.714 & 62 & $\mathrm{~S} 1$ \\
\hline 3718.723 & 14.12 .2005 & 0.720 & 65 & $\mathrm{~S} 1$ \\
\hline 3718.728 & 14.12 .2005 & 0.730 & 62 & $\mathrm{~S} 1$ \\
\hline 3718.731 & 14.12 .2005 & 0.735 & 64 & $\mathrm{~S} 1$ \\
\hline 3718.734 & 14.12 .2005 & 0.741 & 63 & $\mathrm{~S} 1$ \\
\hline 3718.736 & 14.12 .2005 & 0.745 & 63 & $\mathrm{~S} 1$ \\
\hline 3718.740 & 14.12 .2005 & 0.753 & 63 & $\mathrm{~S} 1$ \\
\hline 3718.742 & 14.12 .2005 & 0.757 & 64 & $\mathrm{~S} 1$ \\
\hline 3718.745 & 14.12 .2005 & 0.762 & 66 & $\mathrm{~S} 1$ \\
\hline 3718.748 & 14.12 .2005 & 0.768 & 68 & $\mathrm{~S} 1$ \\
\hline 3718.751 & 14.12 .2005 & 0.774 & 66 & $\mathrm{~S} 1$ \\
\hline 3718.754 & 14.12 .2005 & 0.780 & 65 & S1 \\
\hline 3718.757 & 14.12 .2005 & 0.785 & 63 & $\mathrm{~S} 1$ \\
\hline 3718.760 & 14.12 .2005 & 0.791 & 63 & $\mathrm{~S} 1$ \\
\hline 3718.763 & 14.12 .2005 & 0.797 & 64 & $\mathrm{~S} 1$ \\
\hline 3718.766 & 14.12 .2005 & 0.803 & 65 & S1 \\
\hline 3718.769 & 14.12 .2005 & 0.808 & 64 & $\mathrm{~S} 1$ \\
\hline 3718.772 & 14.12 .2005 & 0.814 & 58 & $\mathrm{~S} 1$ \\
\hline 3718.776 & 14.12 .2005 & 0.822 & 50 & $\mathrm{~S} 1$ \\
\hline 3718.779 & 14.12 .2005 & 0.828 & 49 & $\mathrm{~S} 1$ \\
\hline 3718.782 & 14.12 .2005 & 0.833 & 43 & $\mathrm{~S} 1$ \\
\hline 3718.785 & 14.12 .2005 & 0.839 & 43 & $\mathrm{~S} 1$ \\
\hline 3718.788 & 14.12 .2005 & 0.845 & 49 & $\mathrm{~S} 1$ \\
\hline 3718.791 & 14.12 .2005 & 0.851 & 50 & $\mathrm{~S} 1$ \\
\hline 3718.794 & 14.12 .2005 & 0.856 & 52 & $\mathrm{~S} 1$ \\
\hline 3718.797 & 14.12 .2005 & 0.862 & 59 & $\mathrm{~S} 1$ \\
\hline 3718.800 & 14.12 .2005 & 0.868 & 64 & $\mathrm{~S} 1$ \\
\hline 3718.803 & 14.12 .2005 & 0.874 & 64 & $\mathrm{~S} 1$ \\
\hline 3718.806 & 14.12 .2005 & 0.879 & 61 & $\mathrm{~S} 1$ \\
\hline 3718.808 & 14.12 .2005 & 0.883 & 61 & $\mathrm{~S} 1$ \\
\hline 3718.811 & 14.12 .2005 & 0.889 & 58 & $\mathrm{~S} 1$ \\
\hline 3718.814 & 14.12 .2005 & 0.895 & 62 & S1 \\
\hline 3718.817 & 14.12 .2005 & 0.900 & 60 & $\mathrm{~S} 1$ \\
\hline 3718.820 & 14.12 .2005 & 0.906 & 57 & $\mathrm{~S} 1$ \\
\hline 3718.825 & 14.12 .2005 & 0.916 & 60 & $\mathrm{~S} 1$ \\
\hline 3718.828 & 14.12 .2005 & 0.922 & 61 & $\mathrm{~S} 1$ \\
\hline 3718.831 & 14.12 .2005 & 0.927 & 54 & $\mathrm{~S} 1$ \\
\hline 3718.833 & 14.12 .2005 & 0.931 & 58 & $\mathrm{~S} 1$ \\
\hline 3718.837 & 14.12 .2005 & 0.939 & 49 & $\mathrm{~S} 1$ \\
\hline 3718.849 & 14.12 .2005 & 0.962 & 60 & $\mathrm{~S} 1$ \\
\hline 3718.852 & 14.12 .2005 & 0.968 & 60 & $\mathrm{~S} 1$ \\
\hline 3718.855 & 14.12 .2005 & 0.973 & 53 & $\mathrm{~S} 1$ \\
\hline 3718.858 & 14.12 .2005 & 0.979 & 58 & $\mathrm{~S} 1$ \\
\hline 3718.861 & 14.12 .2005 & 0.985 & 50 & S1 \\
\hline 3718.864 & 14.12 .2005 & 0.991 & 51 & $\mathrm{~S} 1$ \\
\hline 3718.867 & 14.12 .2005 & 0.996 & 52 & $\mathrm{~S} 1$ \\
\hline 3718.870 & 14.12 .2005 & 0.002 & 57 & $\mathrm{~S} 1$ \\
\hline 3718.873 & 14.12 .2005 & 0.008 & 58 & $\mathrm{~S} 1$ \\
\hline 3718.876 & 14.12 .2005 & 0.014 & 57 & $\mathrm{~S} 1$ \\
\hline
\end{tabular}

\begin{tabular}{|c|c|c|c|c|}
\hline HJD & Date & Phase & $\mathrm{S} / \mathrm{N}$ & Subset \\
\hline 3718.879 & 14.12.2005 & 0.019 & 56 & $\mathrm{~S} 1$ \\
\hline 3718.881 & 14.12 .2005 & 0.023 & 57 & S1 \\
\hline 3718.884 & 14.12.2005 & 0.029 & 53 & S1 \\
\hline 3718.887 & 14.12.2005 & 0.035 & 50 & $\mathrm{~S} 1$ \\
\hline 3718.890 & 14.12 .2005 & 0.041 & 52 & $\mathrm{~S} 1$ \\
\hline 3718.893 & 14.12.2005 & 0.046 & 53 & S1 \\
\hline 3718.904 & 14.12.2005 & 0.067 & 51 & $\mathrm{~S} 1$ \\
\hline 3718.907 & 14.12 .2005 & 0.073 & 59 & S1 \\
\hline 3718.909 & 14.12.2005 & 0.077 & 60 & S1 \\
\hline 3718.912 & 14.12 .2005 & 0.083 & 59 & S1 \\
\hline 3718.915 & 14.12.2005 & 0.088 & 59 & S1 \\
\hline 3718.918 & 14.12.2005 & 0.094 & 61 & $\mathrm{~S} 1$ \\
\hline 3718.921 & 14.12 .2005 & 0.100 & 60 & $\mathrm{~S} 1$ \\
\hline 3718.924 & 14.12 .2005 & 0.106 & 57 & S1 \\
\hline 3718.927 & 14.12 .2005 & 0.112 & 54 & S1 \\
\hline 3718.930 & 14.12.2005 & 0.117 & 56 & S1 \\
\hline 3718.933 & 14.12.2005 & 0.123 & 53 & S1 \\
\hline 3718.936 & 14.12 .2005 & 0.129 & 52 & S1 \\
\hline 3718.939 & 14.12 .2005 & 0.135 & 51 & S1 \\
\hline 3718.942 & 14.12 .2005 & 0.140 & 57 & S1 \\
\hline 3718.945 & 14.12 .2005 & 0.146 & 54 & S1 \\
\hline 3718.948 & 14.12.2005 & 0.152 & 56 & S1 \\
\hline 3718.951 & 14.12 .2005 & 0.158 & 45 & S1 \\
\hline 3718.954 & 14.12 .2005 & 0.163 & 52 & S1 \\
\hline 3718.957 & 14.12 .2005 & 0.169 & 51 & S1 \\
\hline 3718.960 & 14.12 .2005 & 0.175 & 53 & S1 \\
\hline 3718.963 & 14.12.2005 & 0.181 & 48 & S1 \\
\hline 3718.966 & 14.12.2005 & 0.186 & 41 & $\mathrm{~S} 1$ \\
\hline 3718.969 & 14.12 .2005 & 0.192 & 45 & S1 \\
\hline 3718.972 & 14.12 .2005 & 0.198 & 48 & S1 \\
\hline 3718.975 & 14.12.2005 & 0.204 & 49 & $\mathrm{~S} 1$ \\
\hline 3718.999 & 14.12 .2005 & 0.250 & 43 & S1 \\
\hline 3719.002 & 14.12 .2005 & 0.255 & 40 & S1 \\
\hline 3719.004 & 14.12.2005 & 0.259 & 43 & $\mathrm{~S} 1$ \\
\hline 3719.007 & 14.12 .2005 & 0.265 & 52 & S1 \\
\hline 3719.010 & 14.12 .2005 & 0.271 & 46 & S1 \\
\hline 3719.013 & 14.12 .2005 & 0.277 & 45 & S1 \\
\hline 3719.016 & 14.12.2005 & 0.282 & 46 & $\mathrm{~S} 1$ \\
\hline 3719.019 & 14.12 .2005 & 0.288 & 42 & S1 \\
\hline 3720.693 & 16.12 .2005 & 0.500 & 56 & S1 \\
\hline 3720.696 & 16.12.2005 & 0.506 & 59 & $\mathrm{~S} 1$ \\
\hline 3720.699 & 16.12 .2005 & 0.511 & 63 & $\mathrm{~S} 1$ \\
\hline 3720.702 & 16.12.2005 & 0.517 & 64 & S1 \\
\hline 3720.705 & 16.12.2005 & 0.523 & 64 & $\mathrm{~S} 1$ \\
\hline 3720.707 & 16.12 .2005 & 0.527 & 70 & S1 \\
\hline 3720.710 & 16.12 .2005 & 0.533 & 68 & S1 \\
\hline 3720.713 & 16.12 .2005 & 0.538 & 69 & S1 \\
\hline 3720.716 & 16.12.2005 & 0.544 & 69 & S1 \\
\hline 3720.719 & 16.12 .2005 & 0.550 & 70 & $\mathrm{~S} 1$ \\
\hline 3720.722 & 16.12 .2005 & 0.556 & 68 & $\mathrm{~S} 1$ \\
\hline 3720.725 & 16.12 .2005 & 0.561 & 69 & S1 \\
\hline 3720.728 & 16.12.2005 & 0.567 & 73 & S1 \\
\hline 3720.730 & 16.12 .2005 & 0.571 & 73 & $\mathrm{~S} 1$ \\
\hline 3720.733 & 16.12 .2005 & 0.577 & 71 & S1 \\
\hline 3720.736 & 16.12 .2005 & 0.582 & 71 & S1 \\
\hline 3720.742 & 16.12 .2005 & 0.594 & 71 & S1 \\
\hline 3720.745 & 16.12.2005 & 0.600 & 72 & S1 \\
\hline 3720.747 & 16.12 .2005 & 0.604 & 72 & S1 \\
\hline 3720.750 & 16.12.2005 & 0.609 & 74 & S1 \\
\hline 3720.753 & 16.12 .2005 & 0.615 & 74 & S1 \\
\hline 3720.756 & 16.12 .2005 & 0.621 & 75 & S1 \\
\hline
\end{tabular}

Article number, page 15 of 21 


\begin{tabular}{|c|c|c|c|c|c|c|c|c|c|}
\hline HJD & Date & Phase & $\mathrm{S} / \mathrm{N}$ & Subset & HJD & Date & Phase & $\mathrm{S} / \mathrm{N}$ & Subset \\
\hline 3720.759 & 16.12.2005 & 0.627 & 74 & S1 & 3720.943 & 16.12.2005 & 0.980 & 68 & S2 \\
\hline 3720.762 & 16.12 .2005 & 0.632 & 74 & S1 & 3720.946 & 16.12 .2005 & 0.985 & 66 & $\mathrm{~S} 2$ \\
\hline 3720.765 & 16.12 .2005 & 0.638 & 76 & S1 & 3720.949 & 16.12 .2005 & 0.991 & 65 & S2 \\
\hline 3720.768 & 16.12 .2005 & 0.644 & 76 & S1 & 3720.952 & 16.12 .2005 & 0.997 & 62 & $\mathrm{~S} 2$ \\
\hline 3720.771 & 16.12 .2005 & 0.650 & 76 & S1 & 3720.955 & 16.12 .2005 & 0.003 & 63 & S2 \\
\hline 3720.774 & 16.12 .2005 & 0.655 & 76 & S1 & 3720.957 & 16.12 .2005 & 0.006 & 62 & $\mathrm{~S} 2$ \\
\hline 3720.777 & 16.12 .2005 & 0.661 & 76 & S1 & 3720.960 & 16.12 .2005 & 0.012 & 60 & $\mathrm{~S} 2$ \\
\hline 3720.779 & 16.12 .2005 & 0.665 & 76 & S1 & 3720.963 & 16.12 .2005 & 0.018 & 61 & S2 \\
\hline 3720.782 & 16.12 .2005 & 0.671 & 77 & S1 & 3720.966 & 16.12 .2005 & 0.024 & 61 & S2 \\
\hline 3720.785 & 16.12 .2005 & 0.676 & 77 & S1 & 3720.969 & 16.12 .2005 & 0.030 & 63 & S2 \\
\hline 3720.790 & 16.12 .2005 & 0.686 & 78 & S1 & 3720.973 & 16.12 .2005 & 0.037 & 64 & S2 \\
\hline 3720.793 & 16.12 .2005 & 0.692 & 78 & S1 & 3720.975 & 16.12 .2005 & 0.041 & 60 & S2 \\
\hline 3720.796 & 16.12 .2005 & 0.698 & 77 & S1 & 3720.978 & 16.12 .2005 & 0.047 & 62 & S2 \\
\hline 3720.799 & 16.12 .2005 & 0.703 & 77 & S1 & 3720.981 & 16.12 .2005 & 0.053 & 60 & S2 \\
\hline 3720.802 & 16.12 .2005 & 0.709 & 78 & S1 & 3720.984 & 16.12 .2005 & 0.058 & 63 & $\mathrm{~S} 2$ \\
\hline 3720.805 & 16.12 .2005 & 0.715 & 78 & S1 & 3720.987 & 16.12 .2005 & 0.064 & 60 & S2 \\
\hline 3720.808 & 16.12 .2005 & 0.721 & 80 & S1 & 3720.990 & 16.12 .2005 & 0.070 & 60 & $\mathrm{~S} 2$ \\
\hline 3720.811 & 16.12 .2005 & 0.726 & 80 & S1 & 3720.993 & 16.12 .2005 & 0.076 & 62 & S2 \\
\hline 3720.814 & 16.12 .2005 & 0.732 & 79 & S1 & 3720.996 & 16.12 .2005 & 0.081 & 61 & S2 \\
\hline 3720.816 & 16.12 .2005 & 0.736 & 79 & S1 & 3720.999 & 16.12 .2005 & 0.087 & 59 & S2 \\
\hline 3720.819 & 16.12 .2005 & 0.742 & 78 & S1 & 3721.001 & 16.12 .2005 & 0.091 & 57 & S2 \\
\hline 3720.822 & 16.12 .2005 & 0.747 & 78 & S1 & 3721.004 & 16.12 .2005 & 0.097 & 58 & S2 \\
\hline 3720.825 & 16.12 .2005 & 0.753 & 55 & S1 & 3721.007 & 16.12 .2005 & 0.102 & 57 & S2 \\
\hline 3720.830 & 16.12 .2005 & 0.763 & 77 & S1 & 3721.010 & 16.12 .2005 & 0.108 & 53 & S2 \\
\hline 3720.833 & 16.12 .2005 & 0.769 & 79 & S1 & 3721.013 & 16.12 .2005 & 0.114 & 54 & S2 \\
\hline 3720.836 & 16.12 .2005 & 0.774 & 79 & S1 & 3721.016 & 16.12 .2005 & 0.120 & 52 & S2 \\
\hline 3720.839 & 16.12 .2005 & 0.780 & 80 & S1 & 3721.026 & 16.12 .2005 & 0.139 & 53 & S2 \\
\hline 3720.842 & 16.12 .2005 & 0.786 & 79 & S1 & 3721.032 & 16.12 .2005 & 0.150 & 55 & S2 \\
\hline 3720.845 & 16.12 .2005 & 0.792 & 77 & S1 & 3721.035 & 16.12 .2005 & 0.156 & 53 & S2 \\
\hline 3720.848 & 16.12 .2005 & 0.797 & 75 & S1 & 3721.038 & 16.12 .2005 & 0.162 & 53 & S2 \\
\hline 3720.851 & 16.12 .2005 & 0.803 & 74 & S1 & 3721.046 & 16.12 .2005 & 0.177 & 48 & S2 \\
\hline 3720.854 & 16.12 .2005 & 0.809 & 74 & S1 & 3722.683 & 18.12.2005 & 0.318 & 60 & S2 \\
\hline 3720.856 & 16.12 .2005 & 0.813 & 72 & S1 & 3722.686 & 18.12.2005 & 0.324 & 63 & S2 \\
\hline 3720.859 & 16.12 .2005 & 0.818 & 70 & S1 & 3722.689 & 18.12.2005 & 0.330 & 62 & S2 \\
\hline 3720.862 & 16.12 .2005 & 0.824 & 70 & S1 & 3722.692 & 18.12 .2005 & 0.335 & 62 & $\mathrm{~S} 2$ \\
\hline 3720.865 & 16.12 .2005 & 0.830 & 71 & S1 & 3722.695 & 18.12.2005 & 0.341 & 66 & S2 \\
\hline 3720.868 & 16.12 .2005 & 0.836 & 71 & S2 & 3722.698 & 18.12.2005 & 0.347 & 66 & S2 \\
\hline 3720.871 & 16.12 .2005 & 0.841 & 71 & S2 & 3722.701 & 18.12.2005 & 0.353 & 68 & S2 \\
\hline 3720.874 & 16.12 .2005 & 0.847 & 72 & S2 & 3722.703 & 18.12.2005 & 0.357 & 68 & S2 \\
\hline 3720.879 & 16.12 .2005 & 0.857 & 70 & S2 & 3722.708 & 18.12.2005 & 0.366 & 71 & S2 \\
\hline 3720.882 & 16.12 .2005 & 0.863 & 69 & S2 & 3722.711 & 18.12 .2005 & 0.372 & 70 & S2 \\
\hline 3720.884 & 16.12 .2005 & 0.866 & 69 & S2 & 3722.714 & 18.12.2005 & 0.378 & 69 & S2 \\
\hline 3720.887 & 16.12 .2005 & 0.872 & 68 & S2 & 3722.717 & 18.12.2005 & 0.383 & 69 & S2 \\
\hline 3720.890 & 16.12 .2005 & 0.878 & 66 & S2 & 3722.720 & 18.12 .2005 & 0.389 & 73 & S2 \\
\hline 3720.893 & 16.12 .2005 & 0.884 & 66 & S2 & 3722.723 & 18.12.2005 & 0.395 & 73 & S2 \\
\hline 3720.896 & 16.12 .2005 & 0.889 & 65 & S2 & 3722.725 & 18.12.2005 & 0.399 & 74 & S2 \\
\hline 3720.899 & 16.12 .2005 & 0.895 & 66 & S2 & 3722.728 & 18.12.2005 & 0.405 & 77 & S2 \\
\hline 3720.902 & 16.12 .2005 & 0.901 & 64 & S2 & 3722.731 & 18.12 .2005 & 0.410 & 78 & S2 \\
\hline 3720.905 & 16.12 .2005 & 0.907 & 63 & S2 & 3722.734 & 18.12 .2005 & 0.416 & 77 & S2 \\
\hline 3720.908 & 16.12 .2005 & 0.912 & 64 & S2 & 3722.737 & 18.12.2005 & 0.422 & 77 & S2 \\
\hline 3720.911 & 16.12 .2005 & 0.918 & 63 & S2 & 3722.740 & 18.12 .2005 & 0.428 & 80 & S2 \\
\hline 3720.914 & 16.12 .2005 & 0.924 & 64 & S2 & 3722.743 & 18.12 .2005 & 0.433 & 80 & S2 \\
\hline 3720.916 & 16.12 .2005 & 0.928 & 64 & S2 & 3722.746 & 18.12 .2005 & 0.439 & 79 & S2 \\
\hline 3720.919 & 16.12 .2005 & 0.934 & 64 & S2 & 3722.749 & 18.12.2005 & 0.445 & 78 & S2 \\
\hline 3720.922 & 16.12 .2005 & 0.939 & 67 & S2 & 3722.752 & 18.12.2005 & 0.451 & 77 & S2 \\
\hline 3720.926 & 16.12.2005 & 0.947 & 66 & S2 & 3722.755 & 18.12 .2005 & 0.456 & 77 & S2 \\
\hline 3720.928 & 16.12 .2005 & 0.951 & 63 & S2 & 3722.758 & 18.12 .2005 & 0.462 & 75 & S2 \\
\hline 3720.931 & 16.12 .2005 & 0.957 & 62 & S2 & 3722.761 & 18.12 .2005 & 0.468 & 75 & S2 \\
\hline 3720.934 & 16.12 .2005 & 0.962 & 65 & S2 & 3722.764 & 18.12 .2005 & 0.474 & 75 & S2 \\
\hline 3720.937 & 16.12 .2005 & 0.968 & 68 & $\mathrm{~S} 2$ & 3722.767 & 18.12 .2005 & 0.479 & 75 & S2 \\
\hline 3720.940 & 16.12 .2005 & 0.974 & 69 & S2 & 3722.770 & 18.12.2005 & 0.485 & 75 & S2 \\
\hline
\end{tabular}




\begin{tabular}{|c|c|c|c|c|c|c|c|c|c|}
\hline HJD & Date & Phase & $\mathrm{S} / \mathrm{N}$ & Subset & HJD & Date & Phase & $\mathrm{S} / \mathrm{N}$ & Subset \\
\hline 3722.772 & 18.12.2005 & 0.489 & 74 & S2 & 3722.966 & 18.12.2005 & 0.861 & 74 & S2 \\
\hline 3722.775 & 18.12.2005 & 0.495 & 75 & S2 & 3722.969 & 18.12 .2005 & 0.867 & 73 & S2 \\
\hline 3722.778 & 18.12 .2005 & 0.500 & 76 & S2 & 3722.972 & 18.12.2005 & 0.873 & 75 & S2 \\
\hline 3722.781 & 18.12 .2005 & 0.506 & 75 & $\mathrm{~S} 2$ & 3722.975 & 18.12 .2005 & 0.878 & 75 & S2 \\
\hline 3722.784 & 18.12.2005 & 0.512 & 76 & S2 & 3722.978 & 18.12.2005 & 0.884 & 74 & S2 \\
\hline 3722.787 & 18.12 .2005 & 0.518 & 76 & $\mathrm{~S} 2$ & 3722.981 & 18.12 .2005 & 0.890 & 74 & S2 \\
\hline 3722.790 & 18.12 .2005 & 0.523 & 75 & $\mathrm{~S} 2$ & 3722.984 & 18.12 .2005 & 0.896 & 74 & S2 \\
\hline 3722.793 & 18.12 .2005 & 0.529 & 73 & $\mathrm{~S} 2$ & 3722.987 & 18.12.2005 & 0.901 & 75 & S2 \\
\hline 3722.796 & 18.12 .2005 & 0.535 & 68 & $\mathrm{~S} 2$ & 3722.990 & 18.12.2005 & 0.907 & 75 & S2 \\
\hline 3722.799 & 18.12.2005 & 0.541 & 64 & $\mathrm{~S} 2$ & 3722.993 & 18.12 .2005 & 0.913 & 75 & S2 \\
\hline 3722.805 & 18.12.2005 & 0.552 & 69 & $\mathrm{~S} 2$ & 3722.996 & 18.12.2005 & 0.919 & 75 & S2 \\
\hline 3722.808 & 18.12 .2005 & 0.558 & 70 & $\mathrm{~S} 2$ & 3722.998 & 18.12 .2005 & 0.923 & 74 & S2 \\
\hline 3722.811 & 18.12.2005 & 0.564 & 71 & S2 & 3723.001 & 18.12.2005 & 0.928 & 74 & S2 \\
\hline 3722.814 & 18.12 .2005 & 0.570 & 66 & $\mathrm{~S} 2$ & 3723.004 & 18.12 .2005 & 0.934 & 75 & S2 \\
\hline 3722.817 & 18.12.2005 & 0.575 & 72 & S2 & 3723.007 & 18.12 .2005 & 0.940 & 75 & S2 \\
\hline 3722.819 & 18.12 .2005 & 0.579 & 73 & S2 & 3723.010 & 18.12.2005 & 0.946 & 74 & S2 \\
\hline 3722.831 & 18.12 .2005 & 0.602 & 77 & $\mathrm{~S} 2$ & 3723.013 & 18.12.2005 & 0.951 & 74 & S2 \\
\hline 3722.834 & 18.12 .2005 & 0.608 & 77 & S2 & 3723.016 & 18.12.2005 & 0.957 & 74 & S2 \\
\hline 3722.836 & 18.12 .2005 & 0.612 & 78 & $\mathrm{~S} 2$ & 3723.020 & 18.12 .2005 & 0.965 & 73 & S2 \\
\hline 3722.839 & 18.12.2005 & 0.617 & 77 & S2 & 3723.022 & 18.12 .2005 & 0.969 & 72 & S2 \\
\hline 3722.842 & 18.12.2005 & 0.623 & 77 & S2 & 3723.025 & 18.12.2005 & 0.974 & 65 & S2 \\
\hline 3722.845 & 18.12 .2005 & 0.629 & 75 & $\mathrm{~S} 2$ & 3723.028 & 18.12.2005 & 0.980 & 70 & S2 \\
\hline 3722.848 & 18.12.2005 & 0.635 & 76 & S2 & 7011.756 & 20.12 .2014 & 0.097 & 100 & S3 \\
\hline 3722.851 & 18.12.2005 & 0.641 & 78 & $\mathrm{~S} 2$ & 7011.760 & 20.12 .2014 & 0.104 & 98 & S3 \\
\hline 3722.854 & 18.12 .2005 & 0.646 & 77 & S2 & 7011.763 & 20.12 .2014 & 0.110 & 101 & S3 \\
\hline 3722.857 & 18.12.2005 & 0.652 & 78 & S2 & 7011.766 & 20.12 .2014 & 0.116 & 102 & S3 \\
\hline 3722.860 & 18.12 .2005 & 0.658 & 78 & S2 & 7011.769 & 20.12 .2014 & 0.122 & 102 & S3 \\
\hline 3722.862 & 18.12 .2005 & 0.662 & 77 & S2 & 7011.772 & 20.12 .2014 & 0.127 & 101 & S3 \\
\hline 3722.865 & 18.12.2005 & 0.667 & 79 & $\mathrm{~S} 2$ & 7011.775 & 20.12 .2014 & 0.133 & 100 & S3 \\
\hline 3722.868 & 18.12 .2005 & 0.673 & 78 & $\mathrm{~S} 2$ & 7011.778 & 20.12 .2014 & 0.139 & 101 & S3 \\
\hline 3722.871 & 18.12.2005 & 0.679 & 80 & $\mathrm{~S} 2$ & 7011.781 & 20.12 .2014 & 0.145 & 101 & S3 \\
\hline 3722.874 & 18.12.2005 & 0.685 & 79 & S2 & 7011.784 & 20.12 .2014 & 0.150 & 100 & S3 \\
\hline 3722.878 & 18.12.2005 & 0.692 & 80 & S2 & 7011.787 & 20.12 .2014 & 0.156 & 98 & S3 \\
\hline 3722.881 & 18.12.2005 & 0.698 & 79 & S2 & 7011.790 & 20.12 .2014 & 0.162 & 97 & S3 \\
\hline 3722.884 & 18.12 .2005 & 0.704 & 80 & $\mathrm{~S} 2$ & 7012.007 & 20.12 .2014 & 0.578 & 71 & S3 \\
\hline 3722.887 & 18.12 .2005 & 0.710 & 80 & S2 & 7012.010 & 20.12 .2014 & 0.584 & 72 & S3 \\
\hline 3722.890 & 18.12 .2005 & 0.715 & 80 & S2 & 7012.013 & 20.12 .2014 & 0.590 & 66 & S3 \\
\hline 3722.893 & 18.12 .2005 & 0.721 & 80 & S2 & 7012.016 & 20.12 .2014 & 0.596 & 55 & S3 \\
\hline 3722.896 & 18.12.2005 & 0.727 & 81 & S2 & 7012.019 & 20.12 .2014 & 0.601 & 55 & S3 \\
\hline 3722.899 & 18.12 .2005 & 0.733 & 80 & $\mathrm{~S} 2$ & 7012.022 & 20.12 .2014 & 0.607 & 58 & S3 \\
\hline 3722.901 & 18.12 .2005 & 0.736 & 80 & S2 & 7012.026 & 20.12 .2014 & 0.615 & 57 & S3 \\
\hline 3722.904 & 18.12.2005 & 0.742 & 80 & S2 & 7012.029 & 20.12 .2014 & 0.621 & 57 & S3 \\
\hline 3722.907 & 18.12 .2005 & 0.748 & 76 & S2 & 7012.032 & 20.12 .2014 & 0.626 & 47 & S3 \\
\hline 3722.910 & 18.12 .2005 & 0.754 & 78 & S2 & 7012.732 & 21.12 .2014 & 0.969 & 90 & S3 \\
\hline 3722.913 & 18.12 .2005 & 0.759 & 79 & S2 & 7012.735 & 21.12 .2014 & 0.975 & 91 & S3 \\
\hline 3722.916 & 18.12 .2005 & 0.765 & 77 & S2 & 7012.738 & 21.12 .2014 & 0.981 & 91 & S3 \\
\hline 3722.919 & 18.12.2005 & 0.771 & 76 & S2 & 7012.741 & 21.12 .2014 & 0.987 & 93 & S3 \\
\hline 3722.922 & 18.12.2005 & 0.777 & 76 & S2 & 7012.744 & 21.12 .2014 & 0.992 & 94 & S3 \\
\hline 3722.925 & 18.12 .2005 & 0.783 & 76 & S2 & 7012.747 & 21.12 .2014 & 0.998 & 94 & S3 \\
\hline 3722.928 & 18.12.2005 & 0.788 & 76 & S2 & 7012.750 & 21.12 .2014 & 0.004 & 94 & S3 \\
\hline 3722.931 & 18.12 .2005 & 0.794 & 76 & S2 & 7012.753 & 21.12 .2014 & 0.010 & 94 & S3 \\
\hline 3722.934 & 18.12 .2005 & 0.800 & 76 & S2 & 7012.756 & 21.12 .2014 & 0.015 & 95 & S3 \\
\hline 3722.937 & 18.12 .2005 & 0.806 & 77 & S2 & 7012.759 & 21.12 .2014 & 0.021 & 93 & S3 \\
\hline 3722.940 & 18.12.2005 & 0.811 & 77 & S2 & 7012.762 & 21.12 .2014 & 0.027 & 95 & S3 \\
\hline 3722.943 & 18.12 .2005 & 0.817 & 75 & S2 & 7012.765 & 21.12 .2014 & 0.033 & 95 & S3 \\
\hline 3722.946 & 18.12 .2005 & 0.823 & 75 & S2 & 7012.973 & 21.12 .2014 & 0.432 & 89 & S3 \\
\hline 3722.949 & 18.12 .2005 & 0.829 & 76 & S2 & 7012.976 & 21.12 .2014 & 0.438 & 87 & S3 \\
\hline 3722.951 & 18.12.2005 & 0.832 & 75 & $\mathrm{~S} 2$ & 7012.979 & 21.12 .2014 & 0.443 & 84 & S3 \\
\hline 3722.954 & 18.12 .2005 & 0.838 & 75 & S2 & 7012.982 & 21.12 .2014 & 0.449 & 88 & S3 \\
\hline 3722.957 & 18.12.2005 & 0.844 & 75 & S2 & 7012.986 & 21.12 .2014 & 0.457 & 87 & S3 \\
\hline 3722.963 & 18.12 .2005 & 0.855 & 75 & S2 & 7012.989 & 21.12 .2014 & 0.462 & 88 & S3 \\
\hline
\end{tabular}




\begin{tabular}{|c|c|c|c|c|c|c|c|c|c|}
\hline HJD & Date & Phase & $\mathrm{S} / \mathrm{N}$ & Subset & HJD & Date & Phase & $\mathrm{S} / \mathrm{N}$ & Subset \\
\hline 7012.992 & 21.12.2014 & 0.468 & 89 & S3 & 7021.015 & 29.12.2014 & 0.862 & 86 & S3 \\
\hline 7012.995 & 21.12.2014 & 0.474 & 83 & S3 & 7021.018 & 29.12.2014 & 0.868 & 85 & S3 \\
\hline 7012.998 & 21.12 .2014 & 0.480 & 81 & S3 & 7021.685 & 30.12 .2014 & 0.148 & 95 & S3 \\
\hline 7013.001 & 21.12.2014 & 0.486 & 83 & S3 & 7021.688 & 30.12 .2014 & 0.153 & 93 & S3 \\
\hline 7013.772 & 22.12 .2014 & 0.965 & 84 & S3 & 7021.691 & 30.12 .2014 & 0.159 & 94 & S3 \\
\hline 7013.775 & 22.12 .2014 & 0.971 & 89 & S3 & 7021.694 & 30.12 .2014 & 0.165 & 97 & S3 \\
\hline 7013.778 & 22.12 .2014 & 0.976 & 90 & S3 & 7021.697 & 30.12 .2014 & 0.171 & 97 & S3 \\
\hline 7013.781 & 22.12.2014 & 0.982 & 90 & S3 & 7021.703 & 30.12 .2014 & 0.182 & 97 & S3 \\
\hline 7013.784 & 22.12 .2014 & 0.988 & 93 & S3 & 7021.706 & 30.12 .2014 & 0.188 & 96 & S3 \\
\hline 7013.787 & 22.12 .2014 & 0.994 & 95 & S3 & 7021.709 & 30.12 .2014 & 0.194 & 97 & S3 \\
\hline 7013.791 & 22.12.2014 & 0.001 & 93 & S3 & 7021.712 & 30.12 .2014 & 0.199 & 99 & S3 \\
\hline 7013.794 & 22.12 .2014 & 0.007 & 95 & S3 & 7021.715 & 30.12 .2014 & 0.205 & 97 & S3 \\
\hline 7013.797 & 22.12 .2014 & 0.013 & 96 & S3 & 7021.719 & 30.12 .2014 & 0.213 & 99 & S3 \\
\hline 7013.800 & 22.12 .2014 & 0.019 & 96 & S3 & 7021.928 & 30.12 .2014 & 0.614 & 100 & S3 \\
\hline 7013.803 & 22.12 .2014 & 0.024 & 95 & S3 & 7021.931 & 30.12 .2014 & 0.620 & 101 & S3 \\
\hline 7013.806 & 22.12 .2014 & 0.030 & 92 & S3 & 7021.934 & 30.12 .2014 & 0.625 & 100 & S3 \\
\hline 7014.013 & 22.12 .2014 & 0.427 & 86 & S3 & 7021.937 & 30.12 .2014 & 0.631 & 100 & S3 \\
\hline 7014.016 & 22.12 .2014 & 0.433 & 83 & S3 & 7021.940 & 30.12 .2014 & 0.637 & 99 & S3 \\
\hline 7014.019 & 22.12 .2014 & 0.439 & 82 & S3 & 7021.943 & 30.12 .2014 & 0.643 & 101 & S3 \\
\hline 7014.022 & 22.12 .2014 & 0.445 & 81 & S3 & 7021.946 & 30.12 .2014 & 0.648 & 101 & S3 \\
\hline 7014.025 & 22.12.2014 & 0.450 & 84 & S3 & 7021.950 & 30.12 .2014 & 0.656 & 100 & S3 \\
\hline 7014.028 & 22.12 .2014 & 0.456 & 84 & S3 & 7021.953 & 30.12 .2014 & 0.662 & 101 & S3 \\
\hline 7014.031 & 22.12 .2014 & 0.462 & 84 & S3 & 7021.956 & 30.12 .2014 & 0.668 & 99 & S3 \\
\hline 7014.034 & 22.12 .2014 & 0.468 & 81 & S3 & 7021.959 & 30.12 .2014 & 0.673 & 101 & S3 \\
\hline 7014.038 & 22.12 .2014 & 0.475 & 77 & S3 & 7021.962 & 30.12 .2014 & 0.679 & 101 & S3 \\
\hline 7014.041 & 22.12 .2014 & 0.481 & 75 & S3 & 7029.733 & 07.01 .2015 & 0.589 & 60 & S4 \\
\hline 7014.044 & 22.12 .2014 & 0.487 & 74 & S3 & 7029.736 & 07.01 .2015 & 0.595 & 61 & S4 \\
\hline 7019.867 & 28.12.2014 & 0.659 & 98 & S3 & 7029.739 & 07.01 .2015 & 0.601 & 62 & S4 \\
\hline 7019.870 & 28.12.2014 & 0.665 & 94 & S3 & 7029.742 & 07.01 .2015 & 0.607 & 59 & S4 \\
\hline 7019.873 & 28.12 .2014 & 0.671 & 99 & S3 & 7029.745 & 07.01 .2015 & 0.612 & 61 & S4 \\
\hline 7019.876 & 28.12.2014 & 0.677 & 96 & S3 & 7029.748 & 07.01 .2015 & 0.618 & 64 & S4 \\
\hline 7019.879 & 28.12 .2014 & 0.682 & 98 & S3 & 7029.751 & 07.01 .2015 & 0.624 & 62 & S4 \\
\hline 7019.882 & 28.12.2014 & 0.688 & 100 & S3 & 7029.755 & 07.01 .2015 & 0.632 & 68 & S4 \\
\hline 7019.885 & 28.12.2014 & 0.694 & 99 & S3 & 7029.758 & 07.01 .2015 & 0.637 & 71 & S4 \\
\hline 7019.889 & 28.12.2014 & 0.702 & 98 & S3 & 7029.761 & 07.01 .2015 & 0.643 & 75 & S4 \\
\hline 7019.892 & 28.12.2014 & 0.707 & 85 & S3 & 7029.764 & 07.01 .2015 & 0.649 & 82 & S4 \\
\hline 7019.895 & 28.12.2014 & 0.713 & 97 & S3 & 7029.767 & 07.01 .2015 & 0.655 & 86 & S4 \\
\hline 7019.898 & 28.12.2014 & 0.719 & 97 & S3 & 7029.973 & 07.01 .2015 & 0.050 & 80 & S4 \\
\hline 7019.901 & 28.12.2014 & 0.725 & 79 & S3 & 7029.976 & 07.01 .2015 & 0.056 & 78 & S4 \\
\hline 7020.746 & 29.12 .2014 & 0.346 & 97 & S3 & 7029.979 & 07.01 .2015 & 0.061 & 75 & S4 \\
\hline 7020.749 & 29.12.2014 & 0.352 & 97 & S3 & 7029.982 & 07.01 .2015 & 0.067 & 77 & S4 \\
\hline 7020.752 & 29.12.2014 & 0.357 & 96 & S3 & 7029.985 & 07.01 .2015 & 0.073 & 74 & S4 \\
\hline 7020.755 & 29.12.2014 & 0.363 & 96 & S3 & 7029.988 & 07.01 .2015 & 0.079 & 71 & S4 \\
\hline 7020.758 & 29.12.2014 & 0.369 & 96 & S3 & 7029.991 & 07.01 .2015 & 0.084 & 71 & S4 \\
\hline 7020.761 & 29.12 .2014 & 0.375 & 97 & S3 & 7029.994 & 07.01 .2015 & 0.090 & 72 & S4 \\
\hline 7020.764 & 29.12 .2014 & 0.380 & 95 & S3 & 7029.997 & 07.01 .2015 & 0.096 & 75 & S4 \\
\hline 7020.767 & 29.12.2014 & 0.386 & 95 & S3 & 7030.000 & 07.01 .2015 & 0.102 & 68 & S4 \\
\hline 7020.770 & 29.12.2014 & 0.392 & 94 & S3 & 7030.004 & 07.01 .2015 & 0.109 & 71 & S4 \\
\hline 7020.773 & 29.12.2014 & 0.398 & 94 & S3 & 7030.007 & 07.01 .2015 & 0.115 & 66 & S4 \\
\hline 7020.776 & 29.12.2014 & 0.403 & 93 & S3 & 7030.692 & 08.01 .2015 & 0.429 & 98 & S4 \\
\hline 7020.780 & 29.12 .2014 & 0.411 & 94 & S3 & 7030.695 & 08.01 .2015 & 0.435 & 97 & S4 \\
\hline 7020.984 & 29.12.2014 & 0.803 & 92 & S3 & 7030.698 & 08.01 .2015 & 0.441 & 98 & S4 \\
\hline 7020.988 & 29.12.2014 & 0.810 & 93 & S3 & 7030.701 & 08.01 .2015 & 0.447 & 97 & S4 \\
\hline 7020.991 & 29.12.2014 & 0.816 & 93 & S3 & 7030.704 & 08.01 .2015 & 0.452 & 97 & S4 \\
\hline 7020.994 & 29.12.2014 & 0.822 & 91 & S3 & 7030.707 & 08.01 .2015 & 0.458 & 97 & S4 \\
\hline 7020.997 & 29.12 .2014 & 0.828 & 91 & S3 & 7030.710 & 08.01 .2015 & 0.464 & 97 & S4 \\
\hline 7021.000 & 29.12.2014 & 0.833 & 88 & S3 & 7030.713 & 08.01 .2015 & 0.470 & 99 & S4 \\
\hline 7021.003 & 29.12 .2014 & 0.839 & 88 & S3 & 7030.717 & 08.01 .2015 & 0.477 & 97 & S4 \\
\hline 7021.006 & 29.12 .2014 & 0.845 & 85 & S3 & 7030.720 & 08.01 .2015 & 0.483 & 98 & S4 \\
\hline 7021.009 & 29.12.2014 & 0.851 & 86 & S3 & 7030.723 & 08.01 .2015 & 0.489 & 98 & S4 \\
\hline 7021.012 & 29.12 .2014 & 0.856 & 85 & S3 & 7030.726 & 08.01 .2015 & 0.495 & 98 & S4 \\
\hline
\end{tabular}




\begin{tabular}{|c|c|c|c|c|}
\hline HJD & Date & Phase & $\mathrm{S} / \mathrm{N}$ & Subset \\
\hline 7030.932 & 08.01 .2015 & 0.890 & 88 & S4 \\
\hline 7030.935 & 08.01.2015 & 0.896 & 90 & S4 \\
\hline 7030.938 & 08.01 .2015 & 0.901 & 89 & S4 \\
\hline 7030.941 & 08.01 .2015 & 0.907 & 89 & S4 \\
\hline 7030.945 & 08.01.2015 & 0.915 & 90 & S4 \\
\hline 7030.948 & 08.01 .2015 & 0.921 & 88 & S4 \\
\hline 7030.951 & 08.01 .2015 & 0.926 & 88 & S4 \\
\hline 7030.954 & 08.01 .2015 & 0.932 & 87 & S4 \\
\hline 7030.957 & 08.01 .2015 & 0.938 & 87 & S4 \\
\hline 7030.960 & 08.01.2015 & 0.944 & 88 & S4 \\
\hline 7030.963 & 08.01 .2015 & 0.949 & 88 & S4 \\
\hline 7030.966 & 08.01 .2015 & 0.955 & 86 & S4 \\
\hline 7031.689 & 09.01.2015 & 0.342 & 95 & S4 \\
\hline 7031.692 & 09.01 .2015 & 0.348 & 94 & S4 \\
\hline 7031.695 & 09.01.2015 & 0.354 & 94 & S4 \\
\hline 7031.698 & 09.01 .2015 & 0.360 & 87 & S4 \\
\hline 7031.701 & 09.01 .2015 & 0.365 & 92 & S4 \\
\hline 7031.704 & 09.01.2015 & 0.371 & 93 & S4 \\
\hline 7031.707 & 09.01 .2015 & 0.377 & 91 & S4 \\
\hline 7031.710 & 09.01.2015 & 0.383 & 92 & S4 \\
\hline 7031.713 & 09.01 .2015 & 0.388 & 91 & S4 \\
\hline 7031.716 & 09.01 .2015 & 0.394 & 93 & S4 \\
\hline 7031.719 & 09.01.2015 & 0.400 & 94 & S4 \\
\hline 7031.722 & 09.01 .2015 & 0.406 & 95 & S4 \\
\hline 7031.928 & 09.01 .2015 & 0.801 & 90 & S4 \\
\hline 7031.931 & 09.01.2015 & 0.807 & 92 & S4 \\
\hline 7031.934 & 09.01 .2015 & 0.812 & 86 & S4 \\
\hline 7031.937 & 09.01.2015 & 0.818 & 88 & S4 \\
\hline 7031.940 & 09.01.2015 & 0.824 & 89 & S4 \\
\hline 7031.943 & 09.01 .2015 & 0.830 & 89 & S4 \\
\hline 7031.947 & 09.01 .2015 & 0.837 & 85 & S4 \\
\hline 7031.950 & 09.01 .2015 & 0.843 & 89 & S4 \\
\hline 7031.953 & 09.01.2015 & 0.849 & 83 & S4 \\
\hline 7031.956 & 09.01 .2015 & 0.855 & 87 & S4 \\
\hline 7031.959 & 09.01 .2015 & 0.860 & 81 & S4 \\
\hline 7031.962 & 09.01.2015 & 0.866 & 77 & S4 \\
\hline 7032.689 & 10.01 .2015 & 0.261 & 86 & S4 \\
\hline 7032.692 & 10.01 .2015 & 0.267 & 86 & S4 \\
\hline 7032.695 & 10.01 .2015 & 0.273 & 88 & S4 \\
\hline 7032.698 & 10.01 .2015 & 0.278 & 86 & S4 \\
\hline 7032.701 & 10.01 .2015 & 0.284 & 85 & S4 \\
\hline 7032.705 & 10.01 .2015 & 0.292 & 86 & S4 \\
\hline 7032.708 & 10.01 .2015 & 0.298 & 85 & S4 \\
\hline 7032.711 & 10.01 .2015 & 0.303 & 84 & S4 \\
\hline 7032.714 & 10.01 .2015 & 0.309 & 84 & S4 \\
\hline 7032.717 & 10.01 .2015 & 0.315 & 83 & S4 \\
\hline 7032.720 & 10.01 .2015 & 0.321 & 89 & S4 \\
\hline 7032.723 & 10.01 .2015 & 0.326 & 89 & S4 \\
\hline 7032.930 & 10.01 .2015 & 0.723 & 89 & S4 \\
\hline 7032.933 & 10.01 .2015 & 0.729 & 90 & S4 \\
\hline 7032.936 & 10.01 .2015 & 0.735 & 86 & S4 \\
\hline 7032.939 & 10.01 .2015 & 0.741 & 86 & S4 \\
\hline 7032.942 & 10.01 .2015 & 0.747 & 87 & S4 \\
\hline 7032.945 & 10.01 .2015 & 0.752 & 87 & S4 \\
\hline 7032.948 & 10.01 .2015 & 0.758 & 84 & S4 \\
\hline 7032.951 & 10.01 .2015 & 0.764 & 87 & S4 \\
\hline 7032.954 & 10.01 .2015 & 0.770 & 87 & S4 \\
\hline 7032.957 & 10.01 .2015 & 0.775 & 83 & S4 \\
\hline 7032.960 & 10.01 .2015 & 0.781 & 82 & S4 \\
\hline 7032.963 & 10.01 .2015 & 0.787 & 83 & S4 \\
\hline 7034.929 & 12.01 .2015 & 0.559 & 91 & S4 \\
\hline
\end{tabular}




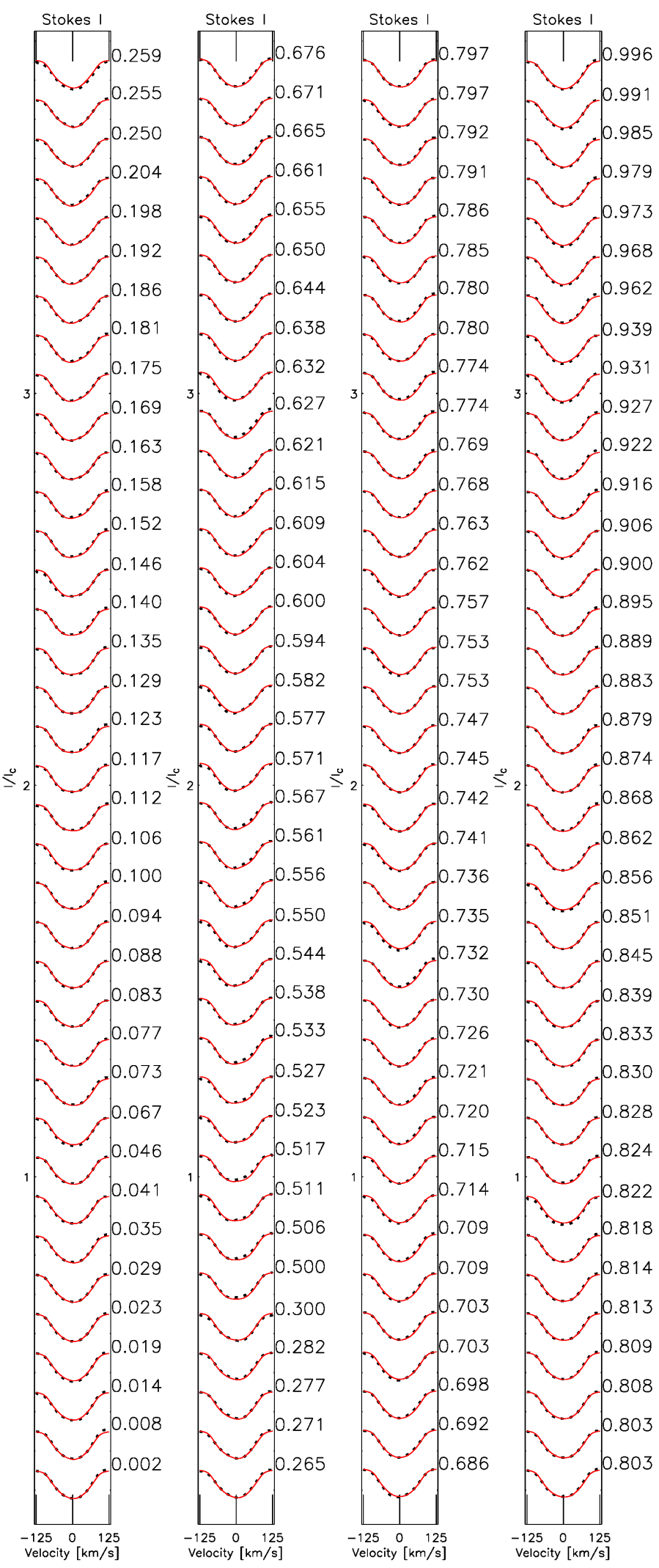

Fig. A.1. Observed line profiles (black dots) and their model fits (red lines) for the S1 Doppler reconstruction shown in Fig. 6. The phases of the individual observations are listed on the right side of the panels.

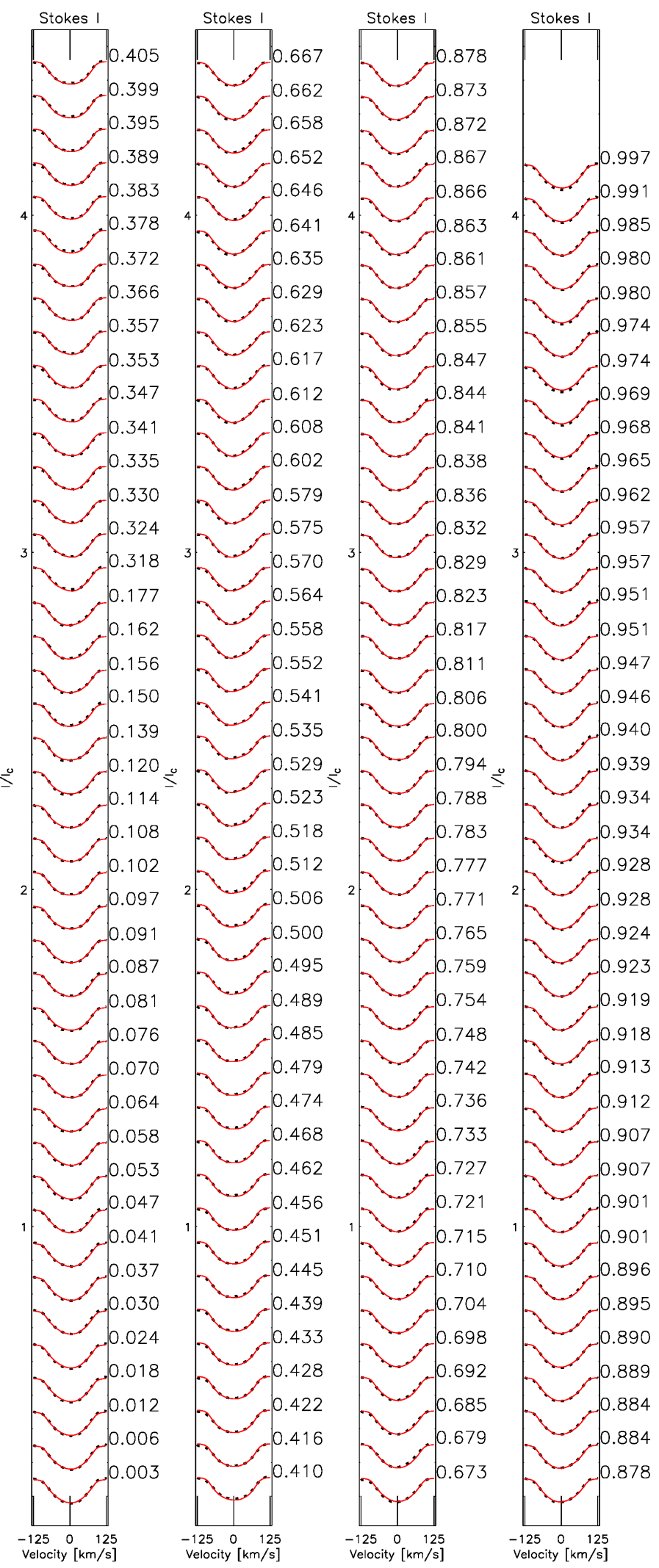

Fig. A.2. Fitted line profiles for the S2 Doppler reconstruction shown in Fig.6 Otherwise as in Fig. A.1 


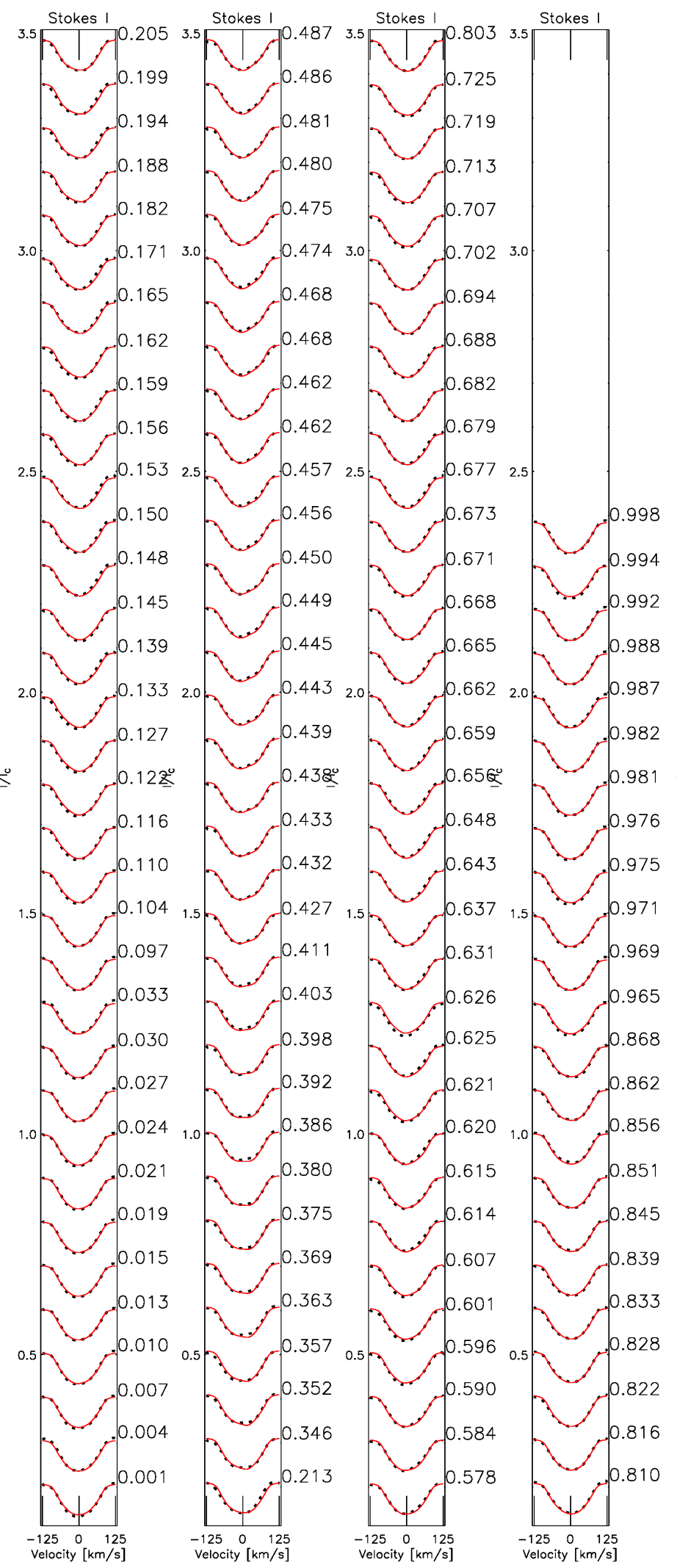

Fig. A.3. Fitted line profiles for the S3 Doppler reconstruction shown in Fig.8 Otherwise as in Fig. A.1

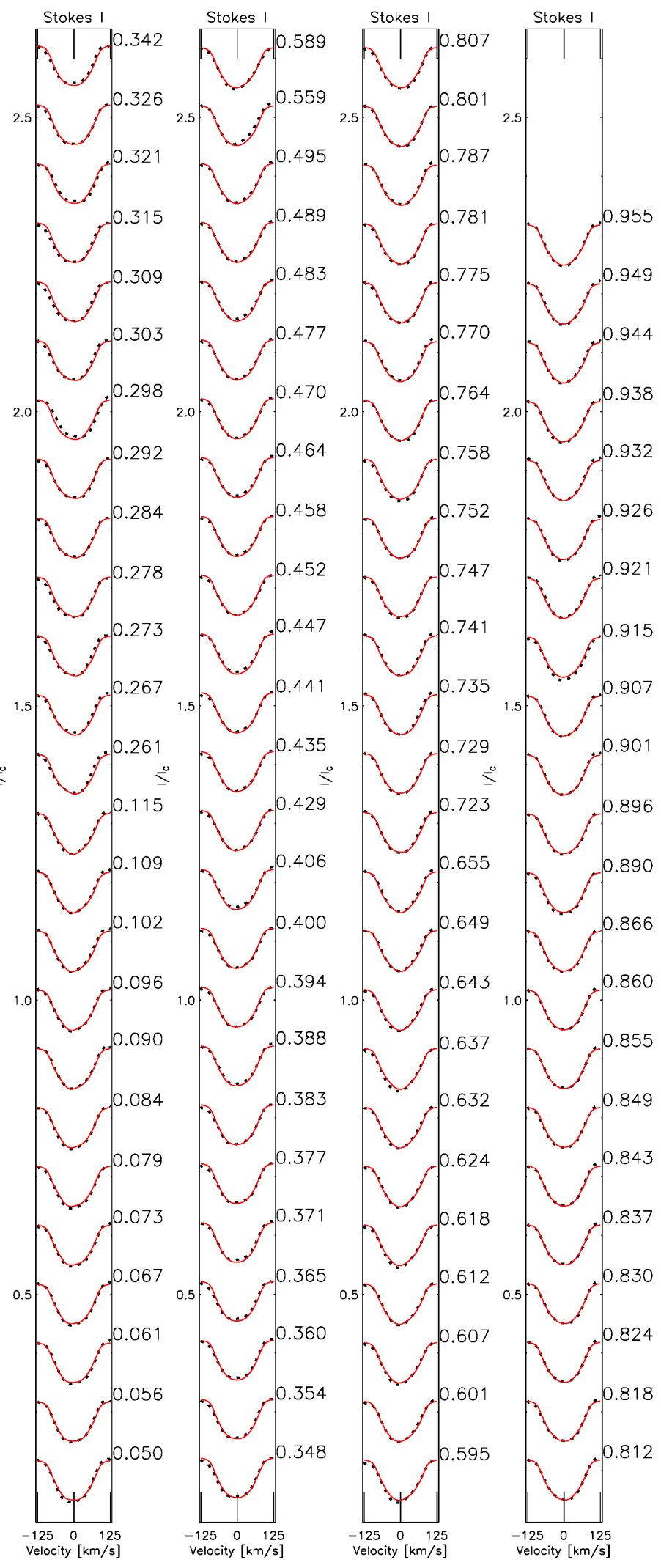

Fig. A.4. Fitted line profiles for the S4 Doppler reconstruction shown in Fig. 8. Otherwise as in Fig. A.1 